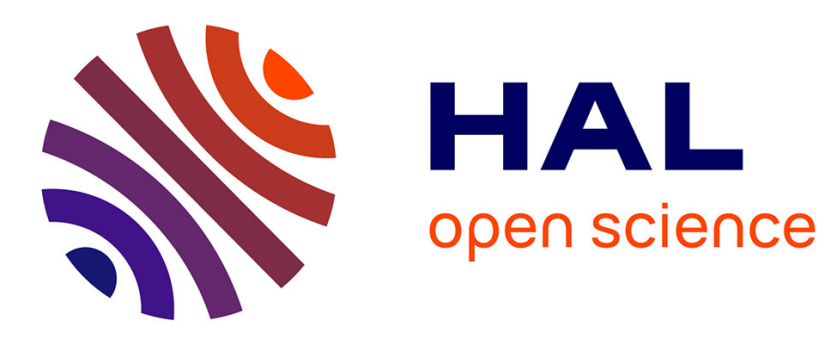

\title{
Overstability and resonance
}

Augustin Fruchard, Reinhard Schäfke

\section{To cite this version:}

Augustin Fruchard, Reinhard Schäfke. Overstability and resonance. Annales de l'Institut Fourier, 2003, 53 (1), pp.227-264. hal-00144874

\section{HAL Id: hal-00144874 \\ https://hal.science/hal-00144874}

Submitted on 6 May 2007

HAL is a multi-disciplinary open access archive for the deposit and dissemination of scientific research documents, whether they are published or not. The documents may come from teaching and research institutions in France or abroad, or from public or private research centers.
L'archive ouverte pluridisciplinaire HAL, est destinée au dépôt et à la diffusion de documents scientifiques de niveau recherche, publiés ou non, émanant des établissements d'enseignement et de recherche français ou étrangers, des laboratoires publics ou privés. 


\title{
Overstability and resonance
}

\author{
Augustin Fruchard and Reinhard Schäfke
}

November 29, 2000

\begin{abstract}
We consider the linear differential equation $\varepsilon y^{\prime \prime}+\varphi(x, \varepsilon) y^{\prime}+\psi(x, \varepsilon) y=0$ where $\varepsilon>0$ is a small parameter and where $x \varphi(x, 0)>0$ if $x \neq 0$. We suppose that $\varphi$ and $\psi$ are real analytic on $[a, b] \times\{0\}$ and that the function $\psi_{0}: x \mapsto \psi(x, 0)$ has a zero at $x=0$ of at least the same order as $\varphi_{0}: x \mapsto \varphi(x, 0)$. We call resonant solution a (family of) solution $y_{\varepsilon}:[a, b] \rightarrow \mathbb{R}$ tending uniformly to a non trivial solution of the reduced equation $\varphi(x, 0) y^{\prime}+\psi(x, 0) y=0$ obtained by formally replacing $\varepsilon$ by 0 and such that all its derivatives remain bounded as $\varepsilon$ tends to 0 . We prove that the existence of a formal series solution whose coefficients have no poles at $x=0$ is a necessary and sufficient condition for the equation to have a resonant solution. This generalizes a result of C.H. Lin. Our proof is based on the study of overstable solutions of a corresponding Riccati equation. The main tool is a "principle of analytic continuation" for overstable solutions of analytic ordinary differential equations of first order, which is also of independent interest.
\end{abstract}

Key words: Resonance, canard solution, overstability, singular perturbation.

ASMS Classification: 34E.

\section{Introduction.}

Originally, the problem of resonance posed by Ackerberg and O'Malley [1] concerned a boundary value problem

$$
\begin{gathered}
\varepsilon y^{\prime \prime}+\varphi(x, \varepsilon) y^{\prime}+\psi(x, \varepsilon) y=0 \\
y(a)=1, \quad y(b)=B>0
\end{gathered}
$$

where $y$ is a real valued function of a real variable $x \in[a, b], \varphi$ and $\psi$ are sufficiently smooth and $\varepsilon>0$ is a small parameter. This problem generally has a unique solution $y(x, \varepsilon)$ and its asymptotic behavior as $\varepsilon \rightarrow 0$ has to be studied.

In case the function $\varphi_{0}: x \mapsto \varphi(x, 0)$ has a constant sign on $[a, b]$, say positive, a boundary layer appears at $x=a$ and on the rest of the interval, $y(x, \varepsilon)$ tends to the solution of the so-called reduced equation

$$
\varphi(x, 0) y^{\prime}+\psi(x, 0) y=0
$$


satisfying the condition $y_{0}(b)=B$. If the sign of $\varphi_{0}$ is negative, the boundary layer appears at $x=b$.

If the function $\varphi_{0}$ vanishes at some point $\left.x_{0} \in\right] a, b[$ - for simplicity, we suppose that $x_{0}=0$ - and if $x \varphi(x, 0) \leq 0$, then, generically, two boundary layers appear at $a$ and $b$ and the solution tends to 0 in the open interval. It can happen, however, that there is only one boundary layer and $y(x, \varepsilon)$ tends to a non trivial solution $y_{0}$ of the reduced equation (3) in the remaining interval (in the sense of uniform convergence on compact subsets of the remaining half-open interval). In this case, we say that (1) exhibits resonance in the sense of Ackerberg-O'Malley.

N. Kopell [14] showed that this problem is equivalent to finding a solution of (1) tending to a non trivial solution of the reduced equation which might not necessarily satisfy the boundary conditions.

In the case that $\varphi_{0}^{\prime}(x)<0$, i.e. $\varphi_{0}$ has a simple turning point in $x=0$, W.D. Lakin [15] and L.P. Cook - W. Eckhaus [10] have shown independently that a necessary condition for resonance is the existence of a formal solution $\hat{y}(x, \varepsilon)=\sum_{n>0} y_{n}(x) \varepsilon^{n}$ of (1) having coefficients $y_{n}$ that are analytic in a neighborhood of $x=0$. This condition had been proposed by Matkowsky [17]; so it is called Matkowsky condition. We would also like to mention the work of Olver [18] and de Groen [11] in this context.

In [19], Y. Sibuya reduces the problem to $\varphi_{0}(x)=-2 x$ and shows that the Matkowsky condition is sufficient for resonance if $\psi$ is analytic with respect to $x$ in a disk with center 0 containing $[a, b]$ (and analytic with respect to $\varepsilon$ near $\varepsilon=0$ ). This condition was improved by C.H. Lin [16]: The Matkowsky condition is sufficient in the case that both $\varphi$ and $\psi$ are analytic in a neighborhood of $[a, b] \times\{0\}$.

We are mainly interested in the case of a turnig point of higher order. Then the Matkowsky condition might not be necessary: In this context, we present a simple example of a linear first order equation whose formal solution has poles at $x=0$ but for which a canard solution exists nevertheless. This led us to strengthen the notion of resonance: We require that all derivatives of $y$ are uniformly bounded. In the present work, we need the additional hypothesis that $\psi_{0}$ has a zero at $x=0$, too, and that its order is at least that of $\varphi_{0}$ at $x=0$. Then we show that the Matkowsky condition is necessary and sufficient for resonance.

Our method of proof is based on two ideas. First, we study a related Riccati equation. More precisely, the transformation $\frac{y}{y^{\prime}}=-\frac{\psi_{0}}{\varphi_{0}}+u$ yields an equation of the form

$$
\varepsilon u^{\prime}=f(x) u+\varepsilon P(x, u, \varepsilon)
$$

where $f=-\varphi_{0}$ and $P$ is analytic ( cf. (26)). Equation (4) is of slow-fast type and hence the theory of canard solutions [4] applies. The precise relation between canard solutions of (4) and resonant solutions of (1) is presented in section 4. This connection had already been established by J.L. Callot in his thesis [7]. In order to simplify matters for readers unfamiliar with nonstandard analysis, we present statements and some proofs in "standard" notation.

The second idea is to consider the problem in the complex domain; this is done not only with respect to $x$, but also with respect to $\varepsilon$. In fact, several general results have been obtained recently $[5,6,9]$ for slow-fast differential equations in the complex domain. Some of them will be presented briefly in section 2 .

Even if the result for a simple turning point is already known, we think that our method is simpler than C.H. Lin's and also we obtain a slightly better result. The case of 
a simple turning point is also treated in a recent article $[5,6]$, where a control parameter is introduced. This method is somewhat related to Kopell's article [14].

The proof of the equivalence between the Matkowsky condition and resonance is given in section 4. As a preparation, we establish an analogous theorem for nonlinear differential equations of first order. This result is also of independent interest in the theory of overstability and canard solutions. As a consequence, the existence of a local canard solution of an analytic first order differential equation implies the existence of a global canard solution.

At the end of the article, we present a construction of an analytic equation of the form (1) satisfying the Matkowsky condition such that all non zero formal solutions diverge. In fact, one could be led to believe that resonance appears only if the formal solutions are convergent (up to a multiplicative factor); there seems to be no simple example where the Matkowsky condition is satisfied, but the formal solutions diverge. Our construction also shows that the general result concerning the Gevrey-1 character of the formal solution is optimal. Thus, we hope to convince some readers, that the problem of resonance is not imaginary.

A part of this work (the proof of theorem 10 in section 3.4) was first carried out in the framework of nonstandard analysis and then translated in standard terms. Here, the hypotheses concerning the smoothness of equation (1) can be significantly weakened. On the other hand, we insist that analyticity with respect to $x$ and $\varepsilon$ is a key hypothesis in the proof of theorem 6 .

\section{Overstability for a non linear first order equation.}

Consider the analytic slow-fast equation

$$
\varepsilon u^{\prime}=\phi(x, u, \varepsilon)
$$

where $x \in[a, b] \subset \mathbb{R}, u \in \mathbb{R}$, and $\varepsilon>0$ is a small parameter (in this article, all derivatives are taken with respect to the variable $x$ ).

We are interested in the asymptotic behavior as $\varepsilon \rightarrow 0$ of families of solutions of (5) depending upon $\varepsilon$. In order to simplify notation and discussion, we talk of solutions instead of families of solutions and often, we omit the $\varepsilon$-dependence. In our context, a solution bounded on an interval $I \subset[a, b]$ is a function defined and bounded on a rectangle of the form $\left.I \times] 0, \varepsilon_{0}\right], \varepsilon_{0}>0$ such that for every $\varepsilon, x \mapsto u(x, \varepsilon)$ is a solution of (5) for that value $\varepsilon$ of the parameter. Note that no regularity condition with respect to $\varepsilon$ is required. We call slow curve a solution $u_{0}=u_{0}(x)$ of the reduced equation $0=\phi(x, u(x), 0)$.

We suppose that the interval $] a, b[$ contains 0 and that equation (5) admits a slow curve that is attractive for $x<0$ and repulsive for $x>0$. We will show that canard solutions having bounded derivatives in a neighborhood of 0 exist if and only if the equation has a formal solution whose coefficients have no singularity at $x=0$. To that purpose, we will use so-called overstable solutions of (5); here (5) is to be considered in the complex domain. More precisely, we will first show that a necessary and sufficient condition for (5) to have an overstable solution is the existence of a formal solution with coefficients analytic at $x=0$, and then we will establish a correspondence between overstable solutions and (real valued) canard solutions. 
We suppose that the above slow curve is $u_{0} \equiv 0$ and the reduced equation is $0=f(x) u$, where $f(x)$ is the partial derivative $f(x)=\frac{\partial \phi}{\partial u}(x, 0,0)$; this can always be achieved by a simple change of variables $u=u_{0}(x)+\varepsilon \tilde{u}(x)$. In this way, we obtain an equation of the form (4).

\subsection{Statement of the principal result.}

We consider equation (4) which we rewrite stating precise hypotheses

$$
\varepsilon u^{\prime}=f(x) u+\varepsilon P(x, u, \varepsilon),
$$

where $f$ and $P$ have the following properties.

- The function $f$ is analytic in a neighborhood (in $\mathbb{C}$ ) of the real interval $[a, b], a<$ $0<b$, and real valued on the real axis.

- We have $x f(x)>0$ for all $x \in[a, b] \backslash\{0\}$; moreover there exists $\lambda>0$ and $p \in 2 \mathbb{N}+1$ such that $f(x)=\lambda x^{p}(1+\mathcal{O}(x))$ as $x \rightarrow 0$.

- The function $P$ is analytic in a neighborhood of $[a, b] \times\{0\} \times\{0\}$ in $\mathbb{C}^{3}$.

Definition 1. - A local canard solution is a (real valued) solution of (6) that is defined and bounded on some interval $]-\delta, \delta[, \delta>0$ in the sense of the beginning of this section, i.e. bounded on some rectangle $\left.]-\delta, \delta[\times] 0, \varepsilon_{0}\right], \delta, \varepsilon_{0}>0$.

A global canard solution is a solution of (6) defined and bounded on the interval $[a, b]$. $u$ is called local, resp. global, $S^{\infty}$ canard solution if for any $n \in \mathbb{N}$, the derivative $u^{(n)}$ exists and is bounded on some interval $]-\delta, \delta[, \delta>0$, resp. on $[a, b]$.

Definition 2. - Let $D \subset \mathbb{C}$ be a simply connected domain containing 0 and $S=$ $S(\alpha, \beta)=\left\{\varepsilon \in \mathbb{C}^{*} \mid \alpha<\arg (\varepsilon)<\beta\right\}$ be a sector. A solution $u=u(x, \varepsilon)$ is called overstable on $D \times S$, if for any domain $D^{\prime}$ whose closure is a compact subset of $D$, there exists some $\varepsilon_{0}>0$ such that the solution $u(x, \varepsilon)$ is defined and bounded on $D^{\prime} \times\{\varepsilon \in S$, $\left.|\varepsilon|<\varepsilon_{0}\right\}$.

We say that $u$ is a local overstable solution in direction $d$ if there exists a neighborhood $D$ of 0 (in $\mathbb{C}$ ) and a sector $S$ containing $e^{d i}$ such that $u$ is overstable on $D \times S$.

We say that $u$ is a global overstable solution in direction $d$ if there exists a neighborhood $D$ of $[a, b]$ (in $\mathbb{C}$ ) and a sector $S$ containing $e^{d i}$ such that $u$ is overstable on $D \times S$.

Remarks: 1 - The sector $S$ in the definition is an infinite sector, but we only need $\varepsilon$ sufficiently close to 0 .

2 - Note that the solution $u$ might not be defined on the whole domain $D$. In the (original) context of nonstandard analysis, an overstable solution was defined and "limited" on the "S-interior" of $D$ for infinitely small $\varepsilon$ in the sector $S$.

3 - Taking the special form of (6) into account, it is natural only to consider canard solutions and overstable solutions close to $u \equiv 0$ as we will do in the sequel.

4 - As was the case for the canard solutions, no regularity with respect to $\varepsilon$ is required. In particular, if for any direction $d$ there is an overstable solution in $D \times S$, where $S$ contains $d$ then these solutions can simply be combined to an overstable solution on $D \times \mathbb{C}^{*}$. On the other hand, an analytic dependence of $\varepsilon$ is, in general, only possible in sectors of angular opening smaller than $\pi$.

The principal result for first order equations in this work is the following. 
Theorem 3. - With the preceding hypotheses and notation, the following statements are equivalent:

1) There exists a formal solution $\hat{u}(x, \varepsilon)=\sum_{n \geq 1} u_{n}(x) \varepsilon^{n}$ of $(6)$ whose coefficients $u_{n}$ are analytic near $x=0$.

2) There exists a local overstable solution of (6) in direction $d$ for every $d$.

3) There exists a global overstable solution of (6) in direction $d=0$.

4) There exists a global $S^{\infty}$ canard solution of (6).

5) There exists a local $S^{\infty}$ canard solution of $(6)$.

Remarks: 1 - The most interesting implications in our opinion are 1) and 5$) \Longrightarrow 4$ ). It seems that the latter implication cannot be proved without using complex analysis. See also example 1 below.

2 - Statement 3) can be improved and made more precise: For every $\delta>0$ there is a neighborhood $D$ of $[a, b]$ and an overstable solution of (6) on $D \times S\left(-\frac{\pi}{2}+\delta, \frac{\pi}{2}-\delta\right)$ analytic with respect to $x$ and $\varepsilon$ (cf theorem 6 ).

3 - The existence of a local overstable solution in one direction $d$ already implies statement 1. This could be proved similarly to 5$) \Longrightarrow 1$ ), but a shorter proof will be given at the end of section 3.1 .

\subsection{Examples.}

Before proving the theorem, we present two simple counterexamples (if the hypotheses are not satisfied). The first is an equation (non analytic with respect to $\varepsilon$ ) having a formal solution without poles but no canard solution. The second example is an equation admitting a canard solution ( not $S^{\infty}$ ) but no formal solution.

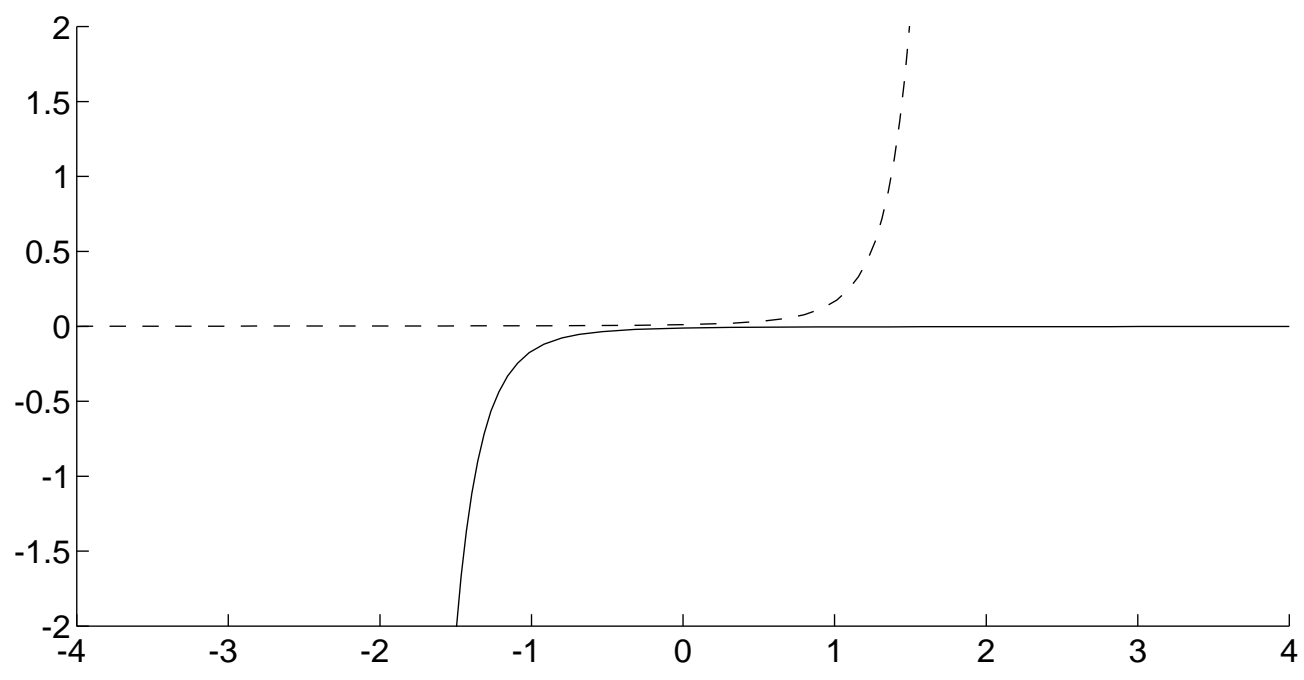

Figure 1: The two solutions of $\varepsilon u^{\prime}=x u+\alpha$ vanishing as $x \rightarrow+\infty$ and $x \rightarrow-\infty$ for $\varepsilon=1 / 4$ and $\alpha=\exp (-1 / \varepsilon)$. 
EXAmPLE 1. - Consider the equation

$$
\varepsilon u^{\prime}=x u+\alpha
$$

where $\alpha$ might depend upon $\varepsilon$ but is constant with respect to $x$. The general solution can be written

$$
u(x)=u\left(x_{0}\right) \exp \left(\frac{x^{2}-x_{0}^{2}}{2 \varepsilon}\right)+\int_{x_{0}}^{x} \frac{\alpha}{\varepsilon} \exp \left(\frac{x^{2}-\xi^{2}}{2 \varepsilon}\right) d \xi .
$$

If the asymptotic expansion of $\alpha=\alpha(\varepsilon)$ as $\varepsilon$ tends to 0 is the zero series, then equation (7) has a formal solution without poles: The zero series. On the other hand, if $\varepsilon \log \alpha(\varepsilon)$ tends to a limit $l$ then the equation has a "boundary" ("butée" [4]) in the points $x= \pm \sqrt{2 l}$. For example, if $\alpha=\exp \left(-\frac{1}{\sqrt{\varepsilon}}\right)$ then there are no canard solutions, if $\alpha=\exp \left(-\frac{1}{\varepsilon}\right)$ then there are local canard solutions but no global canard solutions whenever $a<-\sqrt{2}$ and $\sqrt{2}<b$. The above theorem 3 states that there are no such "boundaries" for differential equations analytic in $x$ and $\varepsilon$ (if a formal solution without poles exists).

Example 2. - Consider

$$
\varepsilon u^{\prime}=2 x^{3} u-2 \varepsilon x .
$$

This equation does not have a formal solution whose coefficients are analytic at $x=0$; its first term is $u_{1}(x)=\frac{1}{x^{2}}$. On the other hand, we will see that it has a canard solution.
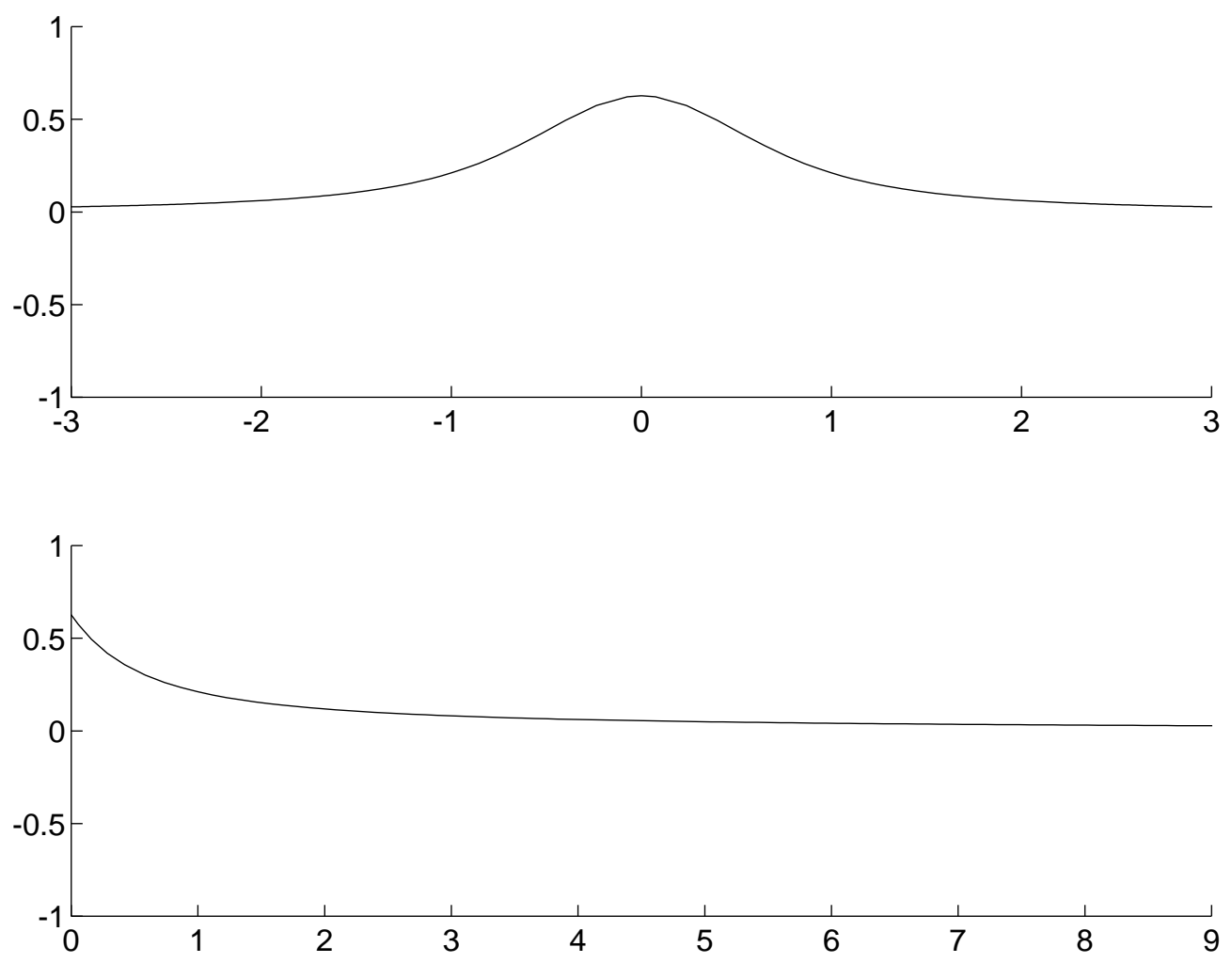

Figure 2: The solutions $\tilde{u}$ and $\tilde{v}$ for $\varepsilon=1 / 4$.

To see this, make a change of variables $t=x^{2}$ and let $v(t)=u(x)$. This leads to the equation

$$
\varepsilon \frac{d v}{d t}=t v-\varepsilon .
$$


Consider now its solution $\tilde{v}$ with initial condition $\tilde{v}(+\infty)=0$. It is defined on $\mathbb{R}$ and given again by variation of constants. Hence

$$
\tilde{v}(t)=\int_{0}^{+\infty} \exp \left(-\frac{s^{2}}{2 \varepsilon}-\frac{t s}{\varepsilon}\right) d s
$$

(One can also write $\tilde{v}$ in terms of the normalised complementary error function $\operatorname{erfcx}(\xi):=$ $e^{\xi^{2}} \int_{\xi}^{+\infty} e^{-u^{2}} d u$; namely $\tilde{v}(t)=\sqrt{2 \varepsilon} \operatorname{erfcx}\left(\frac{t}{\sqrt{2 \varepsilon}}\right)$.) For $t \geq 0$, we have $|\tilde{v}(t)| \leq \tilde{v}(0)=\sqrt{\frac{\pi \varepsilon}{2}}$. Thus the solution $\tilde{u}$ of $(9)$ with initial condition $\tilde{u}(+\infty)=0$, which is given by $\tilde{u}(x)=$ $\tilde{v}\left(x^{2}\right)$, is bounded - and even tends to 0 uniformly - on $\mathbb{R}$.

Note, however, that this solution $\tilde{u}$ has an unbounded fourth derivative and is therefore no $S^{\infty}$ canard solution. Indeed, by differentiating (9) we obtain

$$
\varepsilon u^{(4)}=2 x^{3} u^{(3)}+18 x^{2} u^{\prime \prime}+36 x u^{\prime}+12 u,
$$

hence $\tilde{u}^{(4)}(0)=6 \sqrt{\frac{2 \pi}{\varepsilon}}$.

In the complex plane, the so-called "landscape" [5,8] corresponding to $(9)$ is the surface $\mathbb{C} \times \mathbb{R} \ni(x, z)$ described by $z=R(x):=\operatorname{Re}\left(\int_{0}^{x} 2 \xi^{3} d \xi\right) ;$ here it has four mountains containing each one of the half axes - positive or negative real or imaginary — and four valleys in between. The solution $\tilde{u}$ has, as analytical continuation, a solution close to the slow curve on the two mountains containing the real axis, but on the other two mountains, it is unbounded. An overstable solution would be close to the slow curve on a complete neighborhood of 0 .
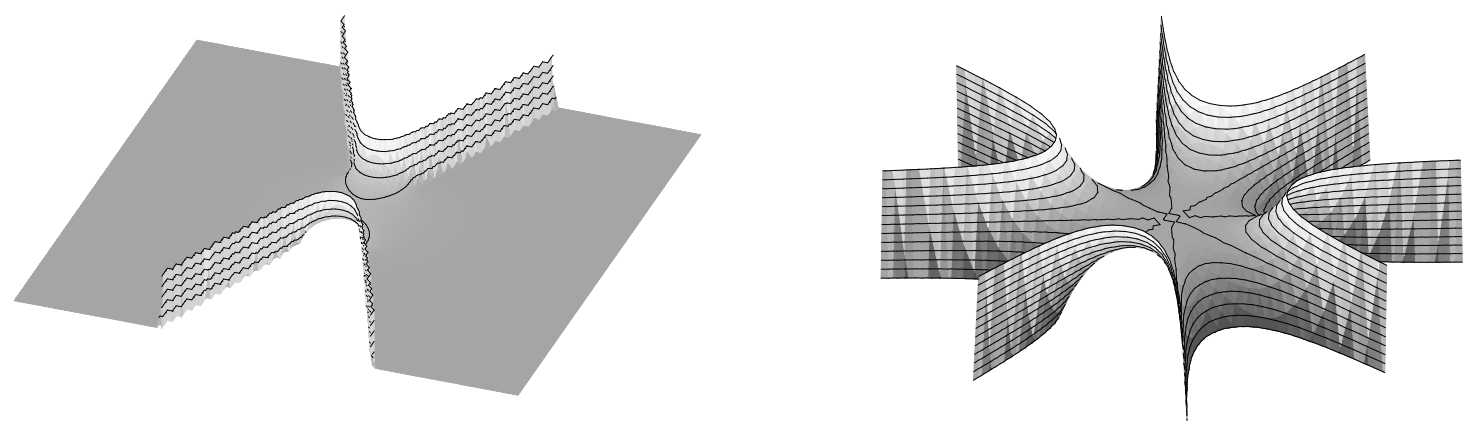

Figure 3: On the left, the absolute value of $\tilde{u}$, on the right, the landscape described by $z=R(x):=\frac{1}{2} \operatorname{Re}\left(x^{4}\right)$.

\section{Proof of theorem 3.}

We will prove that each of the statements implies the following one and that 5$) \Rightarrow 1$ ), but first, we describe the formal solution of (6). These considerations are needed in section 3.5 , but might be of interest already here. 


\subsection{The formal solution of (6).}

The computation of formal solutions $\hat{u}(x, \varepsilon)=\sum_{n \geq 1} u_{n}(x) \varepsilon^{n}$ of $(6)$ having coefficients analytic at $x=0$ yields the following equation

$$
\sum_{n \geq 1} u_{n}^{\prime}(x) \varepsilon^{n+1}=f(x) \sum_{n \geq 1} u_{n}(x) \varepsilon^{n}+\varepsilon \sum_{k, l \geq 0} p_{k, l}(x)\left(\sum_{\nu \geq 1} u_{\nu}(x) \varepsilon^{\nu}\right)^{k} \varepsilon^{l}
$$

where $p_{k, l}$ are defined by $P(x, u, \varepsilon)=\sum_{k, l \geq 0} p_{k, l}(x) u^{k} \varepsilon^{l}$. If $\phi_{n}(x)=\phi_{n}\left(u_{0}, \ldots, u_{n}\right)(x)$ denotes the coefficient of $\varepsilon^{n}$ in the expression

$$
\sum_{k, l \geq 0} p_{k, l}(x)\left(\sum_{\nu \geq 1} u_{\nu} \varepsilon^{\nu}\right)^{k} \varepsilon^{l}
$$

we obtain the following recursion formula (with $u_{0} \equiv 0$ )

$$
\forall n \geq 1, \quad u_{n-1}^{\prime}=f u_{n}+\phi_{n-1} .
$$

If $u_{1}, \ldots, u_{n-1}$ could already be calculated and have no poles at $x=0$ and if

$$
u_{n-1}^{\prime}(x)-\phi_{n-1}(x)=\mathcal{O}\left(x^{p}\right)
$$

as $x \rightarrow 0$ then $(12)$ yields $u_{n}:=\frac{1}{f}\left(u_{n-1}^{\prime}-\phi_{n-1}\right)$ which is analytic near $x=0$. Conversely, condition (13) is clearly a necessary condition for $u_{n}$ to be analytic near $x=0$. Thus we have shown

Proposition 4. - There is a formal solution of (6) whose coefficients $\left(u_{n}\right)_{n \in \mathbb{N}}$ are analytic near $x=0$ if and only if the order of the zero $x=0$ of $u_{n}^{\prime}-\phi_{n}\left(u_{0}, \ldots, u_{n}\right)$ is at least $p$ for every integer $n$. In this case, the coefficients are given recursively by $u_{0}=0$ and

$$
u_{n}=\frac{1}{f}\left(u_{n-1}^{\prime}-\phi_{n-1}\right) \text {. }
$$

Note that for every $n \geq 1$, if $u_{1}, \ldots, u_{n-1}$ could already be calculated and have no poles at $x=0$ then there is a function $R_{n}(x, \varepsilon)$ analytic and bounded in a neighborhood of $x=0$ (in the sense of the beginning of section 2) such that

$$
P\left(x, \sum_{\nu=1}^{n-1} u_{\nu}(x) \varepsilon^{\nu}, \varepsilon\right)=\sum_{\nu=0}^{n-1} \phi_{\nu}(x) \varepsilon^{\nu}+R_{n}(x, \varepsilon) \varepsilon^{n} .
$$

This will be useful later.

On this occasion we give a proof of the statement in remark 3 below theorem 3 . Suppose that $u$ is a local overstable solution in one direction $d$. We show that $u$ has an asymptotic expansion $u(x, \varepsilon) \sim \sum_{n=0}^{\infty} u_{n}(x) \varepsilon^{n}$ as $\varepsilon \rightarrow 0$ in direction $d$, i.e. for every $n$ we show the existence of analytic $u_{1}(x), \ldots, u_{n-1}(x)$ and of $v_{n}(x, \varepsilon)$ analytic and bounded in some neighborhood of $x=0$ and for $\varepsilon$ in some sector containing direction $d$, such that

$$
u(x, \varepsilon)=\sum_{\nu=1}^{n-1} u_{\nu}(x) \varepsilon^{\nu}+v_{n}(x, \varepsilon) \varepsilon^{n}
$$


This of course implies the existence of a formal solution.

We proceed by induction. For $n=0$ there is nothing to prove. For $n=1$ we use $f(x) u(x, \varepsilon)=\varepsilon u^{\prime}(x, \varepsilon)-\varepsilon P(x, u(x, \varepsilon), \varepsilon)$; first this shows that $f(x) \frac{1}{\varepsilon} u(x, \varepsilon)$ is bounded in some neighborhood of the origin (using Cauchy's formula) and hence also $\frac{1}{\varepsilon} u(x, \varepsilon)$ is bounded in some neighborhood of 0 if we exclude a small disk around 0 , then the boundedness of $\frac{1}{\varepsilon} u(x, \varepsilon)$ on a full neighborhood of 0 follows by the maximum principle.

Suppose now that statement (16) is true for some $n$. Using (15), insertion into (6) implies

$$
f(x) v_{n}(x, \varepsilon)=\left(u_{n-1}^{\prime}-\phi_{n-1}\right)(x)+\varepsilon R_{n}(x, \varepsilon) .
$$

As $f(x)$ has a zero of order $p$ at $x=0$, Rouché's theorem applied to small circles around 0 implies that $u_{n-1}^{\prime}-\phi_{n-1}$ also has a zero at least of order $p$ at $x=0$ and hence $u_{n}=\frac{1}{f}\left(u_{n-1}^{\prime}-\right.$ $\left.\phi_{n-1}\right)$ is analytic in some full neighborhood of 0 . Thus $f(x)\left(v_{n}(x, \varepsilon)-u_{n}(x)\right)=\varepsilon R_{n}(x, \varepsilon)$ and as before $v_{n+1}(x, \varepsilon)=\frac{1}{\varepsilon}\left(v_{n}(x, \varepsilon)-u_{n}(x)\right)$ is bounded in some neighborhood of 0 .

\subsection{From the formal solution to overstable solutions.}

In this section, we begin the proof of theorem 3 . Concerning the implication 1$) \Rightarrow 2$ ), we simply use a general result of [9] (proposition 8.3), which can be expressed in terms of the present article in the following way.

Proposition 5. - If equation (6) admits a formal solution whose coefficients are analytic near $x=0$ (and hence on $[a, b]$ ) then it has a local overstable solution for any direction $d$.

A direct proof could use so-called modified Nagumo norms [9], construct a majorant equation (without derivatives) and would first conclude that the formal solution is of Gevrey type (i.e. its coefficients grow at most like factorials). Then the Borel transform of this series converges locally and its truncated Laplace transform yields a quasi-solution, i.e. a function satisfying (6) except for an exponentially small error. Finally Gronwall's lemma would provide an overstable solution. We refer to [9] for more information.

Another proof would imbed (6) in an equation with $p$ additional parameters

$$
\varepsilon u^{\prime}=f(x) u+\varepsilon P(x, u, \varepsilon)+\sum_{k=1}^{p} \alpha_{k} x^{k-1}
$$

and use the result 6 of [6]. Let $F$ be the antiderivative of $f$ vanishing at $x=0$ and let $C_{r}$ be the connected component containing 0 of the set $\{x \in \mathbb{C} ;|F(x)|<r\}$. For sufficiently small $r$, the functions $f$ and $P$ are analytic in a neighborhood of the closure of $C_{r}$, resp. $C_{r} \times\{0\} \times\{0\}$. By the cited result, for every direction $d$ and $\varepsilon$ with arg $\varepsilon=d$, summation "to the least term" $\bar{a}=\sum_{\nu=0}^{[r / \varepsilon]} a_{\nu} \varepsilon^{\nu}$ of the unique formal solution $\hat{a}=\sum_{n \geq 0} a_{n} \varepsilon^{n}, \hat{u}=$ $\sum_{n \geq 0} u_{n}(x) \varepsilon^{n}\left(u_{n}\right.$ analytic on $\left.[a, b]\right)$ provides a value of the parameter $a=\left(\alpha_{1}, \ldots, \alpha_{p}\right)$ for which (17) has an overstable solution in this direction. As it was supposed that (6) without parameter already has a formal solution with analytic coefficients, $\hat{a}$ has to be the zero series; hence $\bar{a}=0$.

In any case, the construction of a local overstable solution from a formal solution needs powerful tools already present in other articles and will not be further detailed here.

Observe that the overstable solution provided by the theorem will in general not depend analytically upon $\varepsilon$ in a full neighborhood of $\varepsilon=0$; this corresponds to the divergence 
of the formal solution. Proposition 8.3 of [9], however, yields overstable solutions that are analytic with respect to $\varepsilon$ in small sectors with vertices at the origin.

\subsection{Analytic continuation of an overstable solution.}

We show here the following result.

Theorem 6. - If equation (6) has an overstable solution in some neighborhood of $x=0$ for every direction $d$ then for any $\delta>0$ there exists a neighborhood $D$ of $[a, b]$ and an analytic overstable solution of $(6)$ on $D \times S\left(-\frac{\pi}{2}+\delta, \frac{\pi}{2}-\delta\right)$.

We again consider the antiderivative $F$ of $f$ vanishing at $x=0$. By the hypotheses on $f$, the function $F$ has real values on the real axis. Furthermore, there are $\tilde{a}, \tilde{b} \in \mathbb{R}$ with $\tilde{a}<a<b<\tilde{b}$ such that $F(\tilde{a}) \neq F(\tilde{b})$ and the functions $f$ and $P$ of $(6)$ are analytic with respect to $x$ in a simply connected domain $D$ containing $[\tilde{a}, \tilde{b}]$. It is therefore sufficient to show the existence of an overstable solution in a domain containing $] \tilde{a}, \tilde{b}[$. Without loss of generality, we can also assume that $F(\tilde{a})>F(\tilde{b})$. For simplicity of notation we will write $a, b$ instead of $\tilde{a}, \tilde{b}$.

The following lemmas seem to be well known. They can, for example, be found in [8] in a version using nonstandard analysis and in [21] in the linear case. We present here statements adapted for use in this article. For the convenience of the reader, we also include proofs.

The first lemma is analogous to theorem 2 of [8]. This result states that a solution of (6) with initial condition near the slow curve can be continued analytically along a path descending the landscape described by $z=R(x):=\operatorname{Re}(F(x))$. We have modified this lemma in the following way: (a) We construct a solution on an entire $x$-domain and not only a restriction to a path. (b) The solution we construct is analytic with respect to $\varepsilon$ in a sector that can be chosen large if the domain permits this. For the sake of simplicity, we will only present the result for a particular kind of $x$-domain.

Lemma 7. - Let $x_{1} \in D$ and consider a $C^{1}$ family $\Gamma=\left\{\gamma_{s} \mid s \in[0,1]\right\}$ of paths starting at $x_{1}$, i.e. a $C^{1}$ mapping $\Gamma:[0,1]^{2} \rightarrow D$ such that $\Gamma(s, 0)=x_{1}$ for all $s \in[0,1]$. Let $d_{1}<d_{2}$ be two directions in $\mathbb{S}^{1}$. Suppose that all the paths in the family $\Gamma$ are downward for every direction in $\left[d_{1}, d_{2}\right]$; in other words,

$$
\forall d \in\left[d_{1}, d_{2}\right], \forall(s, t) \in[0,1]^{2}, \frac{d}{d t} R_{d}\left(\gamma_{s}(t)\right)<0
$$

where $R_{d}(x):=\operatorname{Re}\left(F(x) e^{-i d}\right)$. Denote by $K$ the image $\Gamma\left([0,1]^{2}\right)$ of $\Gamma$. Finally, assume that $u_{1}=u_{1}(\varepsilon)$ is an initial condition analytic in $\varepsilon$ and satisfies $u_{1}=o(1)$ as $\varepsilon \rightarrow 0$.

Then there exists $\varepsilon_{0}>0$ such that the solution $u$ of $(6)$ with initial condition $u\left(x_{1}, \varepsilon\right)=$ $u_{1}(\varepsilon)$ is analytic in a neighborhood of $S \times K$ where $S:=\left\{\varepsilon \in \mathbb{C}|0<| \varepsilon \mid \leq \varepsilon_{0}, d_{1} \leq\right.$ $\left.\arg \varepsilon \leq d_{2}\right\}$ and satisfies $u(x, \varepsilon)=o(1)$ as $S \ni \varepsilon \rightarrow 0$ uniformly on $K$.

Proof. By compactness, there exist $C, \delta>0$ such that for all $(s, t) \in[0,1]^{2}$ and $d \in\left[d_{1}, d_{2}\right]$ one has $\left|\gamma_{s}^{\prime}(t)\right|<C$ and $\frac{d}{d t} R_{d}\left(\gamma_{s}(t)\right)<-\delta$.

By shrinking $D$ somewhat, if necessary, we can assume that for $r, \varepsilon>0$ sufficiently small, the absolute value $|P(x, u, \varepsilon)|$ is bounded by some constant $M$ for $x \in D,|u|<r$ and $|\varepsilon|<\varepsilon_{0}$. 
Let $\rho \in] 0, r\left[\right.$. We show that for sufficiently small $\rho$, there exist $\varepsilon_{1}>0$ such that for every $\varepsilon \in S$ with $|\varepsilon|<\varepsilon_{1}$ and every $s \in[0,1]$, the function $v_{\varepsilon}: t \mapsto u\left(\varepsilon, \gamma_{s}(t)\right)$ is defined on $[0,1]$ and satisfies $\left|v_{\varepsilon}(t)\right|<\rho$. Since $\varepsilon$ is small we have $\left|v_{\varepsilon}(0)\right|=\left|u_{1}(\varepsilon)\right|<\rho$. It is sufficient to show that the set $\left\{\left|v_{\varepsilon}\right|<\rho\right\}$ is invariant under (6), i.e. if $\left|v_{\varepsilon}(t)\right|=\rho$ then $\frac{d}{d t}\left|v_{\varepsilon}(t)\right|<0$ for sufficiently small $\varepsilon$. We calculate

$$
\begin{aligned}
\frac{d}{d t} v(t) \bar{v}(t) & =2 \operatorname{Re}\left(u^{\prime}\left(\gamma_{s}(t)\right) \gamma_{s}^{\prime}(t) \bar{u}\left(\gamma_{s}(t)\right)\right) \\
& =\operatorname{Re}\left(\left(\frac{2}{\varepsilon} f\left(\gamma_{s}(t)\right) v(t)+2 P\left(\gamma_{s}(t), v(t), \varepsilon\right)\right) \gamma_{s}^{\prime}(t) \bar{v}(t)\right) \\
& =\frac{2}{|\varepsilon|}|v(t)|^{2} \frac{d}{d t} R_{d}\left(\gamma_{s}(t)\right)+p(t, v(t), \varepsilon)
\end{aligned}
$$

where $d:=\arg \varepsilon$ and $p(t, v, \varepsilon):=2 \operatorname{Re}\left(P\left(\gamma_{s}(t), v, \varepsilon\right) \gamma_{s}^{\prime}(t) \bar{v}\right)$.

If $|v(t)|=\rho$ then we find $\frac{d}{d t}|v(t)|^{2} \leq-2 \delta \rho^{2} /|\varepsilon|+2 M C \rho$, hence $\frac{d}{d t}|v(t)|^{2}<0$ provided $|\varepsilon|<\varepsilon_{1}:=\min \left(\varepsilon_{0}, \frac{\delta \rho}{M C}\right)$. This proves the existence of $u$ along every path of $\Gamma$ and also that $u(x, \varepsilon)=o(1)$ as $S \ni \varepsilon \rightarrow 0$. The analyticity with respect to $\varepsilon$ comes from the theorem on analytic dependence upon parameters.

The second lemma presented here corresponds essentially to the propositions 1 and 2 and theorem 1 of [8]. This result treats the exponential closeness of solutions of (6). Here again we needed to change the statements somewhat for our needs and to give them a more quantitative meaning than in [8].

Lemma 8. - Let $u_{0}$ be a solution of $(6)$ defined in some simply connected $x$-domain $\Omega$ and for sufficiently small $\varepsilon$ with $\arg \varepsilon=d$. Let $x_{1}$ and $x_{2}$ be two points of $\Omega$ and $\gamma$ a path in $\Omega$ connecting them. Let $u$ be another solution of (6) defined in some neighborhood of $x_{1}$ and let finally $\alpha \geq 0$ such that

$$
u\left(x_{1}, \varepsilon\right)-u_{0}\left(x_{1}, \varepsilon\right)=o\left(e^{-\alpha /|\varepsilon|}\right)
$$

Suppose that for $x \in \gamma([0,1])$ we have $R_{d}(x)-R_{d}\left(x_{1}\right) \leq \alpha$.

Then the solution $u$ can be continued analytically along $\gamma$ and the estimate

$$
u\left(x_{2}, \varepsilon\right)-u_{0}\left(x_{2}, \varepsilon\right)=o\left(e^{-\beta /|\varepsilon|}\right) \quad \text { holds with } \beta=\alpha+R_{d}\left(x_{1}\right)-R_{d}\left(x_{2}\right) .
$$

Proof. We use the fact that the difference $y_{\varepsilon}=u(., \varepsilon)-u_{0}(., \varepsilon)$ satisfies an equation of the form

$$
\varepsilon y_{\varepsilon}^{\prime}=f(x) y_{\varepsilon}+\varepsilon P_{1}\left(x, u_{0}(x), y_{\varepsilon}, \varepsilon\right) y_{\varepsilon}
$$

where $P_{1}$ is some function analytic in a neighborhood of $\Omega \times\{0\}^{3}$ satisfying $P(x, u+$ $y, \varepsilon)-P(x, u, \varepsilon)=P_{1}(x, u, y, \varepsilon) y$ there.

On any path $\mathcal{C}$ containing $x_{1}$ where $y_{\varepsilon}$ is defined and satisfies $\left|y_{\varepsilon}(x)\right| \leq \delta$ with some sufficiently small $\delta$, we conclude that $y_{\varepsilon}$ satisfies

$$
y_{\varepsilon}(x)=y_{\varepsilon}\left(x_{1}\right) \exp \left(\frac{1}{\varepsilon}\left(F(x)-F\left(x_{1}\right)+\mathcal{O}(\varepsilon)\right)\right)=\mathcal{O}\left(y_{\varepsilon}\left(x_{1}\right) \exp \left(\frac{1}{|\varepsilon|}\left(R_{d}(x)-R_{d}\left(x_{1}\right)\right)\right)\right)
$$

uniformly on $\mathcal{C}$ and hence, by the hypotheses of the lemma $y_{\varepsilon}(x)=o(1)$ as $\varepsilon \rightarrow 0$. 
By a classical theorem on the existence of solutions of ordinary differential equations, $y_{\varepsilon}$ can be continued analytically along $\gamma$ and satisfies the estimate

$$
y(x)=o\left(\exp \left(\frac{1}{|\varepsilon|}\left(R_{d}(x)-R_{d}\left(x_{1}\right)-\alpha\right)\right)\right)
$$

on it.

Continuation of the proof of theorem 6. Remember that the meaning of $a$ and $b$ has changed since the beginning of the proof: It is sufficient, at this point, to prove the existence of an overstable solution in some domain containing $] a, b[$.

For $r \in] 0, F(b)$, we denote by $C_{r}$ the connected component of the set $\{x \in D|| F(x) \mid \leq$ $r$ \} containing 0 . If $r$ is sufficiently small, then $C_{r}$ is compact and for every direction $d \in \mathbf{S}^{1}$, there exists an overstable solution $u_{0}^{d}$ in some domain containing $C_{r}$.

Denote by $\frac{\pi}{2}>\delta>\delta^{\prime}>0$ the numbers satisfying $r=F(b) \sin \delta=F(a) \sin \delta^{\prime}($ see figure 4 below). Consider the domain $\Delta$ enclosed by the $\operatorname{arcs}\{x \in D \mid \arg (F(a)-F(x))=$ $\left.\pm \delta^{\prime},|F(a)-F(x)| \leq F(a) \cos \delta^{\prime}\right\}$ and $\{x \in D|\arg (F(b)-F(x))= \pm \delta| F,(b)-F(x) \mid \leq$ $F(b) \cos \delta\}$ and by the two parts of the boundary of $C_{r}$ making these into a Jordan curve, which is $C^{1}$ except in $a$ and $b$. For sufficiently small $r>0$ the closure of this domain is contained in $D$. We have $\Delta=\Delta_{a} \cup \Delta_{b} \cup \tilde{C}_{r}$, where $\Delta_{a}$ and $\Delta_{b}$ are the domains, containing $a$ resp. $b$ on their boundary, whose $F$-images are the triangles with vertices $F(a), p_{a}, q_{a}$ resp. $F(b), p_{b}, q_{b}$, where $p_{a}=r \exp i\left(\pi / 2-\delta^{\prime}\right), q_{a}=r \exp i\left(\delta^{\prime}-\pi / 2\right), p_{b}=$ $r \exp i(\pi / 2-\delta), q_{b}=r \exp i(\delta-\pi / 2)$.
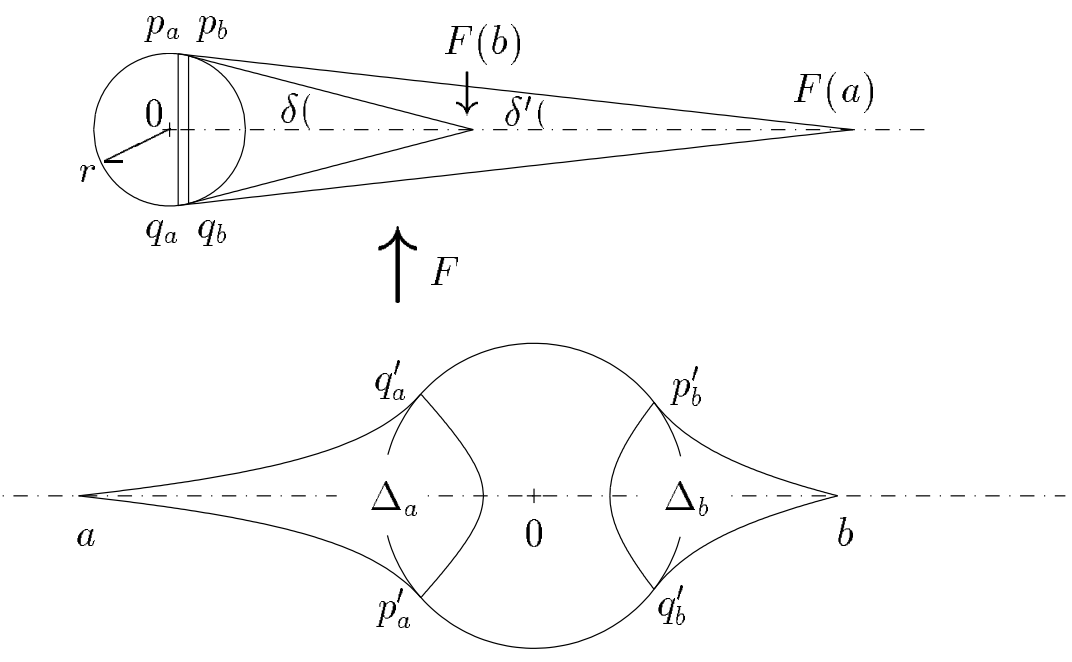

Figure 4: The domain $\Delta$ and its $F$-image (here $F(x)=x^{2}$ ). Primes denote the points whose $F$-images are the points without primes.

We consider now $\varepsilon$ in the closed sector $S_{\delta}=\left\{\varepsilon \in \mathbb{C}|| \arg (\varepsilon) \mid \leq \frac{\pi}{2}-\delta\right\}$ and the solutions $u_{a}$ and $u_{b}$ of (6) vanishing at $x=a$ resp. $x=b$. By lemma 7 (cf. proposition $\mathrm{D}$ of [5]) these solutions are overstable in $\Delta_{a} \times S_{\delta^{\prime}}$ resp. $\Delta_{b} \times S_{\delta}$. As we already have an overstable solution $u_{0}^{d}$ in $C_{r}$ for every direction $d \in \mathbb{S}^{1}$, lemma 8 yields that both $u_{a}$ and $u_{b}$ 
can be continued analytically to overstable solutions on $C_{r} \times S_{\delta^{\prime}}$, resp. $C_{r} \times S_{\delta}$ (cf. also the commentary 'relation entrée-sortie' below proposition $\mathrm{E}$ in [5]). In particular, they are both defined in $x=0$. Moreover, by lemma 8 , we have for every $\tilde{r}<r$

$$
u_{a}(0, \varepsilon)-u_{b}(0, \varepsilon)=\mathcal{O}(\exp (-\tilde{r} / \varepsilon))
$$

for $\varepsilon$ with $|\arg (\varepsilon)| \leq \frac{\pi}{2}-\delta$ and sufficiently small $|\varepsilon|$. Consider now the function $\varphi$ : $\varepsilon \mapsto u_{a}(0, \varepsilon)-u_{b}(0, \varepsilon)$. It is analytic and bounded in the sector $S_{0}=\{\varepsilon \in \mathbb{C}|| \varepsilon \mid<$ $\left.\varepsilon_{0},|\arg (\varepsilon)| \leq \frac{\pi}{2}-\delta\right\}$. Moreover it satisfies, for small $\varepsilon$ with $|\arg (\varepsilon)|=\frac{\pi}{2}-\delta$ the estimate $|\varphi(\varepsilon)|=\mathcal{O}(\exp (-(r+o(1)) /|\varepsilon|)=\mathcal{O}(\exp (-(F(b)+o(1)) / \varepsilon)$. The classical PhragmenLindelöf theorem implies that this estimate holds for all sufficiently small $\varepsilon$ in $S_{0}$. Using again lemma 8 (this time with $u_{0}=u_{b}, u=u_{a}$ ), we conclude that for $\arg \varepsilon \in\left[-\frac{\pi}{2}+\right.$ $\left.\delta, \frac{\pi}{2}-\delta\right]$, the solution $u_{a}$ is overstable on the entire domain $\Delta$. As $\delta$ becomes small with $r \in] 0, F(b)$ [ chosen above, the proof is complete.

Remark: We restricted the theorem to the case that $f$ is real valued on the real axis and $[a, b]$ is a real interval, but theorem 6 can easily be adapted to more "complex" situations.

\subsection{From overstable solutions to canard solutions.}

Proposition 9. - If there exists a local (resp. global) overstable solution of (6) then there also exists a local (resp. global) $S^{\infty}$ canard solution.

Proof. Given a local (resp. global) overstable solution we construct one having real values on the real axis, namely the solution having a certain real initial condition on some point of the real axis and then we use lemma 8. For example, using the notation of the preceding section, if $F(a) \geq F(b)$, the solution vanishing at some point $\tilde{a}<a$ sufficiently close to $a$ is overstable on some neighborhood of $[a, b]$. (by the way, this is the solution called $u_{\tilde{a}}$ of the above proof).

The restriction of such a canard solution to the real axis is obviously a canard solution. It is a $S^{\infty}$ canard solution, because by Cauchy's formula, the derivatives will also be bounded on $[a, b]$.

\subsection{From canard solutions to the formal solution.}

We have already proved all the implications $i) \Rightarrow i+1$ ) of theorem 3 (the last one is trivial). To complete its proof, it is sufficient to show that the last statement implies the first.

Theorem 10. - If equation (6) has a local $S^{\infty}$ canard solution, then it also has a formal solution $\hat{u}(\varepsilon, x)=\sum_{n \geq 1} u_{n}(x) \varepsilon^{n}$ having coefficients analytic in a neighborhood of $x=0$. These are determined recursively by (14).

Proof. We give an indirect proof; so suppose that (6) has a $S^{\infty}$ canard solution $u=u(x, \varepsilon)$ on some interval $[-\delta, \delta]$ independent of $\varepsilon$ but there is no formal solution of $(6)$ without poles at $x=0$. Using proposition 4 , we can suppose that there is some $n \geq 2$ such that

$$
u_{k-1}^{\prime}-\phi_{k-1}=f u_{k} \text { for } k \in\{2, \ldots, n-1\}
$$


(if $n>2$ ) and

$$
u_{n-1}^{\prime}(x)-\phi_{n-1}(x)=-a_{q} x^{q}+x^{q+1} g(x)
$$

with $q<p, a_{q} \neq 0$ and $g$ analytic in some neighborhood of $x=0$. We will show that some derivative $u^{(m)}(x, \varepsilon)$ cannot be bounded as $\varepsilon \rightarrow 0$. So consider the difference between $u$ and the $(n-1)$-th partial sum of the formal solution. Seen under some adequate "magnifying glass", i.e. some change of the dependent and independent variables, this difference is a bounded solution of some regularly perturbed differential equation. Consequently, the dominant term of this solution satisfies a certain differential equation without polynomial solution. Taking into account that the change of variables in some sense "flattens" the derivatives, we deduce that some derivative of sufficiently high order must be unbounded as $\varepsilon \rightarrow 0$. We now give a detailed proof.

We consider the following change of the dependent variable:

$$
u=\sum_{k=1}^{n-1} u_{k} \varepsilon^{k}+\frac{\varepsilon^{n}}{\eta} v \text { with } \eta=\varepsilon^{\frac{p-q}{p+1}} .
$$

Lemma 11. - If $u$ is a solution of (6), then the function $v$ defined by (20) satisfies an ordinary differential equation of the form

$$
\varepsilon v^{\prime}=(f(x)+\varepsilon Q(x, v)) v+\eta\left(a_{q} x^{q}-x^{q+1} g(x)\right)+\varepsilon \eta R(x)
$$

where $g, Q$ and $R$ are analytic for $x$ in some small interval $[-\delta, \delta]$, small $\varepsilon$ and $v$ in some disk, and remain bounded as $\varepsilon \rightarrow 0$.

Proof. Insertion of (20) into (6) yields

$$
\sum_{k=1}^{n-1} u_{k}^{\prime} \varepsilon^{k+1}+\frac{\varepsilon^{n+1}}{\eta} v^{\prime}=\sum_{k=1}^{n-1} f(x) u_{k} \varepsilon^{k}+\frac{\varepsilon^{n}}{\eta} f(x) v+\varepsilon P\left(x, \Sigma u+\frac{\varepsilon^{n}}{\eta} v, \varepsilon\right),
$$

where we write $\Sigma u$ for $\sum_{k=1}^{n-1} u_{k} \varepsilon^{k}$. By using (18) for $k=1, \ldots, n-1$ and multiplying by $\frac{\eta}{\varepsilon^{n}}$, we obtain

$$
\left.\varepsilon v^{\prime}=f(x) v-\eta\left(u_{n-1}^{\prime}(x)-\phi_{n-1}(x)\right)\right)+\frac{\eta}{\varepsilon^{n-1}}\left(P\left(x, \Sigma u+\frac{\varepsilon^{n}}{\eta} v, \varepsilon\right)-\sum_{k=0}^{n-1} \phi_{k}(x) \varepsilon^{k}\right) .
$$

Now

$$
P\left(x, \Sigma u+\frac{\varepsilon^{n}}{\eta} v, \varepsilon\right)=P(x, \Sigma u, \varepsilon)+\frac{\varepsilon^{n}}{\eta} v Q(x, v)
$$

with

$$
Q(x, v):=\int_{0}^{1} \frac{\partial P}{\partial u}\left(x, \Sigma u+\frac{\varepsilon^{n}}{\eta} v \theta, \varepsilon\right) d \theta
$$

Moreover, $P(x, \Sigma u, \varepsilon)=\sum_{k=0}^{n-1} \phi_{k}(x) \varepsilon^{k}+\varepsilon^{n} R(x)$ with $R:=R_{n}$ by formula (15) in section 3.1. This implies the lemma together with formula (19).

We will use now the attractivity of the slow curve to show that $v$ remains bounded as $\varepsilon \rightarrow 0$ on $[-\delta, 0[$. For fixed $x<0$ this follows simply from the existence of an asymptotic expansion away from 0 . ([12] or [20] theorem 40.1). We first show that this persists for some $x=x(\varepsilon)$ of order $\varepsilon^{1 / p+1}$. 
Lemma 12. - For all $r>0$ there exist $\varepsilon_{1}>0$ and $Z>0$ such that $|v(\varepsilon, x)| \leq r$ for all $x<-Z \varepsilon^{1 /(p+1)}$ and $0<\varepsilon \leq \varepsilon_{1}$.

It is sufficient to show this for sufficiently small $r$. We already know that $v(x, \varepsilon)=\mathcal{O}(\eta)$ on every interval of the form $[a,-\delta]$ with $\delta>0$ independent of $\varepsilon$. If $\varepsilon$ is sufficiently small, it remains to show the statement on $\left[-\delta,-Z \varepsilon^{1 /(p+1)}\right.$ [ for some properly chosen $Z$.

By the hypotheses, for $r>0$ sufficiently small, there is $K>0$ such that $|Q(\varepsilon, x, v)| \leq$ $K$ for all $x \leq 0,|v| \leq r$ and small $\varepsilon$. Moreover, there exists $\mu>0$ such that $f(x) \leq-\mu|x|^{p}$ for $-\delta \leq x \leq 0$.

It is sufficient to show that the set $\{|v| \leq r\}$ is an invariant set for (21) on the interval $\left[-\delta,-Z \varepsilon^{1 /(p+1)}\right.$ [, if $Z$ has been chosen properly. It is hence sufficient to show that for $x<-Z \varepsilon^{1 /(p+1)}$, we have $v^{\prime}(\varepsilon, x)<0$ if $v(\varepsilon, x)=r$ (and similarly $v^{\prime}(\varepsilon, x)>0$ if $v(\varepsilon, x)=-r)$.

For $x=-z \varepsilon^{1 /(p+1)}$ such that $v(\varepsilon, x)=r$, we find using $(21)$

$$
\varepsilon v^{\prime}(\varepsilon, x) \leq-\mu r \varepsilon^{p /(p+1)} z^{p}+\left|a_{q}\right| \varepsilon^{p /(p+1)} z^{q}+\mathcal{O}(\varepsilon)
$$

and hence $v^{\prime}(\varepsilon, x)<0$ if $z>Z$ where $Z$ is chosen greater than $\left(\frac{\left|a_{q}\right|}{\mu r}\right)^{1 /(p-q)}$, and if $\varepsilon$ is sufficiently small.

We now make the following change of variables: $x=\varepsilon^{\frac{1}{p+1}} X, V(X, \varepsilon)=v(x, \varepsilon)$ and $f(x)=\varepsilon^{\frac{p}{p+1}} F(X, \varepsilon)$. Thus we have $F(X, \varepsilon)=X^{p}(\lambda+o(1))$ uniformly for $X$ in bounded subsets of the real axis with some $\lambda \neq 0$ independent of $\varepsilon$.

Equation (21) is transformed into

$$
\dot{V}=\lambda X^{p} V+a_{q} X^{q}+\alpha(\varepsilon) \tilde{P}(\varepsilon, X, V),
$$

where the dot denotes differentiation with respect to $X$, where $\alpha(\varepsilon)$ tends to 0 as $\varepsilon$ tends to 0 and $\tilde{P}$ denotes a function analytic and bounded (as $\varepsilon \rightarrow 0$ ) on all bounded $X, V$-sets. Lemma 12 provides an initial condition $X_{0}=X_{0}(\varepsilon)$, negative and bounded, such that $V\left(X_{0}, \varepsilon\right)$ remains bounded. By compactness, there is a sequence $\varepsilon_{\nu} \rightarrow 0$ such that $X_{0}\left(\varepsilon_{\nu}\right)$ has a limit, say $\check{X}$, and $V\left(X_{0}\left(\varepsilon_{\nu}\right), \varepsilon_{\nu}\right)$ has a limit, say $\check{V}$.

Lemma 13. - Suppose that $W$ is the solution of

$$
\dot{W}=\lambda X^{p} W+a_{q} X^{q}
$$

with initial condition $W(\check{X})=\check{V}$. Then for every integer $m \geq 0$ and for every real $X$ we have

$$
V^{(m)}(X, \varepsilon) \rightarrow W^{(m)}(X), \quad \varepsilon \rightarrow 0
$$

Proof. Consider (23) and (24) in the complex domain. As $W$ is a solution of a linear ordinary differential equation without finite singularity, $W$ is an entire function. By the classical theorem on the dependence upon parameters, it follows that $V\left(X, \varepsilon_{\nu}\right)$ is defined for every point $X$ and sufficiently large $\nu$. Moreover, we have $V\left(X, \varepsilon_{\nu}\right) \rightarrow W(X)$, as $\nu \rightarrow 0$, uniformly for $X$ in any bounded subset of $\mathbb{C}$. For the derivatives this follows using Cauchy's formula or the differential equation.

The proof of the following lemma is immediate (compare degrees and use $p>q$ ).

Lemma 14. - Equation (24) has no polynomial solution. 
We return now to the proof of theorem 10. Lemmas 13 and 14 imply that for every positive integer $m$ there are a $X_{m} \in \mathbb{R}$ and $a_{m}>0$ independent of $\varepsilon$ such that $\left|V^{(m)}\left(X_{m}, \varepsilon\right)\right| \geq$ $a_{m}$ for sufficiently small $\varepsilon$ (one could choose $a_{m}:=\frac{1}{2} W^{(m)}\left(X_{m}\right)$ with $W^{(m)}\left(X_{m}\right) \neq 0$ ). We conclude that $\left|v^{(m)}\left(X_{m} \varepsilon^{\frac{1}{p+1}}, \varepsilon\right)\right|=\varepsilon^{\frac{-m}{p+1}}\left|V^{(m)}\left(X_{m}, \varepsilon\right)\right| \geq \varepsilon^{\frac{-m}{p+1}} a_{m}$, hence $u^{(m)}\left(x_{m}\right)=$ $\varepsilon^{n+\frac{q-p-m}{p+1}} \alpha_{m}+\mathcal{O}(1)$ with $\left|\alpha_{m}\right| \geq a_{m}$. Thus, for $m>n(p+1)$, the absolute value of $u^{(m)}\left(x_{m}\right)$ tends to $\infty$ as $\varepsilon \rightarrow 0$, contradicting the hypothesis of the theorem.

\section{Application to linear differential equation of second order.}

In this section, we return to equation (1)

$$
\varepsilon y^{\prime \prime}+\varphi(x, \varepsilon) y^{\prime}+\psi(x, \varepsilon) y=0
$$

where $\varphi$ and $\psi$ are analytic with respect to $(x, \varepsilon)$ in a neighborhood of $[a, b] \times\{0\}$ in $\mathbb{C}^{2}$. As in the introduction, $\varphi_{0}$ and $\psi_{0}$ are obtained from $\varphi$ and $\psi$ by putting $\varepsilon=0$. We suppose that $\varphi_{0}$ has a unique zero in $[a, b]$ and denote by $p$ its order. Moreover, we suppose that $\varphi_{0}$ is positive on $[a, 0[$ and negative on $] 0, b]$ (and hence $p$ is odd).

As before, the derivatives are taken with respect to $x$ and we often omit to indicate the dependence upon $\varepsilon$. The word "bounded" always denotes "bounded uniformly with repect to $\varepsilon$ ". Again the dependence of the solutions upon $\varepsilon$ needs not be analytic unless explicitly stated.

Definition 15. - Let $I \subset[a, b]$ be an interval containing 0 in its interior. Then a $S^{\infty}$ resonant solution on $I$ is a solution of (1) that is bounded on $I$ (in the above sense) that does not tend to the zero function as $\varepsilon \rightarrow 0$ and whose derivatives of any order are bounded on $I$. A local $S^{\infty}$ resonant solution is a $S^{\infty}$ resonant solution on some open interval containing 0 . A global $S^{\infty}$ resonant solution is a $S^{\infty}$ resonant solution on $[a, b]$. A local analytic resonant solution in direction $d$ is a solution of (1) analytic in some neighborhood of $x=0$ that does not tend to the zero function as $\varepsilon \rightarrow 0$ in some sector containing $d$. A global analytic resonant solution in direction $d$ is a solution of (1) analytic on some domain containing $[a, b]$ that does not tend to the zero function as $\varepsilon \rightarrow 0$ in some sector containing $d$.

From this point on, we have to make a rather restrictive hypothesis.

Hypothesis 4 . - The function $\psi_{0}$ has a zero of order $p$ or higher at $x=0$.

If this hypothesis is not satisfied, it seems possible to show that the existence of a resonant solution implies that $\psi_{0}$ has a zero of order $\geq p-1$ and that $-x \psi_{0}(x) / \varphi_{0}(x)$ tends to a nonnegative integer congruent to 0 or $1 \bmod p+1$ as $x \rightarrow 0$. We hope that we can deal with this more general setting in a future article.

Theorem 16. - Under the preceding hypotheses and with the above notation, the following statements are equivalent:

1) There exists a formal solution $\hat{y}(x, \varepsilon)=\sum_{n \geq 0} y_{n}(x) \varepsilon^{n}$ of $(1)$ whose coefficients $y_{n}$ are analytic in a neighborhood of 0 .

2) There exists a local $S^{\infty}$ resonant solution.

3) There exists a global $S^{\infty}$ resonant solution. 
4) There exist local analytic resonant solution for every direction d.

5) There exists a global analytic resonant solution for the direction $d=0$.

Remarks: 1 - The implication 1) $\Rightarrow 3$ ) generalizes C.H.Lin's theorem [16]. The implication 1$) \Rightarrow 5$ ) slightly improves his theorem even in the case of a simple turning point (i.e. $\varphi_{0}$ has a simple zero at $x=0 ; p=1$ ).

2 - Statement 5) can be improved as below theorem 3: for every $\delta>0$ there is a neighborhood $D$ of $[a, b]$ and a global analytic resonant solution of $(6)$ on $D \times S\left(-\frac{\pi}{2}+\delta, \frac{\pi}{2}-\delta\right)$ analytic with respect to $x$ and $\varepsilon$.

3 - If $p=1$ then hypothesis 4 is not needed: It is possible to reduce all situations such that it is satisfied by going over from (1) to a second order equation for $y^{\prime}$ etc.

Proof. As indicated in the introduction, we use the corresponding Riccati equation: We put $v=\frac{y^{\prime}}{y}$ and obtain the following equation

$$
\varepsilon v^{\prime}=-\varphi(x, \varepsilon) v-\psi(x, \varepsilon)-\varepsilon v^{2}
$$

which has the slow curve $v_{0}=-\frac{\psi_{0}}{\varphi_{0}}$. It is at this point that hypothesis 4 is needed; it guarantees that the slow curve is analytic on $[a, b]$. The change of variables $v=v_{0}+u$ yields an equation of the form (6)

$$
\varepsilon u^{\prime}=f(x) u+\varepsilon P(x, u, \varepsilon)
$$

with $f=-\varphi_{0}$ and

$$
P=\frac{\varphi_{0}-\varphi}{\varepsilon} u+\frac{\varphi \psi_{0}-\varphi_{0} \psi}{\varepsilon \varphi_{0}}+\frac{1}{\varphi_{0}^{2}}\left(\varphi_{0}^{\prime} \psi_{0}-\varphi_{0} \psi_{0}^{\prime}\right)-\left(-\frac{\psi_{0}}{\varphi_{0}}+u\right)^{2} .
$$

These functions satisfy the hypotheses 1,2 and 3 of section 2.1 and theorem 3 can be applied. It is hence sufficient to establish a correspondence between the formal solution of (1) and that of (6) as well as one between $S^{\infty}$ resonant solutions of (1) and canard solutions of (6) resp. analytic resonant solutions of (1) and overstable solutions of (6). In other words, theorem 16 relies on the following statement.

Proposition 17. - 1. Equation (1) has a non trivial formal solution $\hat{y}(x, \varepsilon)=$ $\sum_{n \geq 0} y_{n}(x) \varepsilon^{n}$ with coefficients analytic on $[a, b]$ if and only if $(6)$ has a formal solution $\hat{u}(x, \varepsilon)=\sum_{n \geq 1} u_{n}(x) \varepsilon^{n}$ with coefficients analytic on $[a, b]$.

2. Let $y$ be a solution of (1) and let $u=\frac{y^{\prime}}{y}-v_{0}=\frac{y^{\prime}}{y}+\frac{\psi_{0}}{\varphi_{0}}$ be the corresponding solution of (6). Then $y$ is a local (resp. global) $S^{\infty}$ resonant solution of (1) if and only if $u$ is a local (resp. global) $S^{\infty}$ canard solution of (6). Furthermore $y$ is a local (resp. global) analytic resonant solution of (1) if and only if $u$ is a local (resp. global) overstable solution of (6).

Proof. 1. If equation (1) has a non trivial formal solution $\hat{y}(x, \varepsilon)=\sum_{n>0} y_{n}(x) \varepsilon^{n}$ with coefficients analytic on $[a, b]$ then its first term $y_{0}$ is a nontrivial solution of a non singular linear first order equation (divide the reduced equation by $x^{p}$ ) and hence does not vanish on $[a, b]$. It follows that (6) also has a formal solution $\hat{u}=\frac{\hat{y}^{\prime}}{\hat{y}}+\frac{\psi_{0}}{\varphi_{0}}$, whose coefficients are analytic on $[a, b]$. Conversely, if $\hat{u}(x, \varepsilon)=\sum_{n \geq 1} u_{n}(x) \varepsilon^{n}$ is a formal solution of (6) then the expression $\exp \left(\int_{0}^{x} v_{0}(\xi) d \xi\right) \exp \left(\int_{0}^{x} \hat{u}(\xi, \varepsilon) d \xi\right)$ is a formal series that (formally) satisfies (1). 
2. The equivalence between Ackerberg-O'Malley resonance (not in the $S^{\infty}$ sense) of the linear equation (1) and the presence of canard solutions of the associated Riccati equation (25) had been established in [7]. These results of Jean-Louis Callot can also be found in [3].

Also here, $u=\frac{y^{\prime}}{y}-v_{0}$ is equivalent to $y=\exp \left(\int^{x}\left(u+v_{0}\right) d \xi\right)$ and clearly this establishes a correspondance between analytic solutions.

As the solutions $u$ of (6) and $v$ of (25) are related by $u=v-v_{0}$, the statements concerning $u$ are equivalent to those concerning $v$. It remains (for the equivalence of $S^{\infty}$ resonance and $S^{\infty}$ canards) to show that $y^{(n)}$ is bounded for every $n$ if and only if $v^{(n)}$ is bounded for every $n$. To show this, induction yields

$$
v^{(n-1)}=\frac{y^{(n)}}{y}-\frac{P_{n}\left(y, y^{\prime}, \ldots, y^{(n-1)}\right)}{y^{n}}
$$

where $P_{n}$ is a polynomial. As $y_{0}$ does not vanish on $[a, b]$ this shows that " $y$ (n) are bounded for all $n$ " implies " $v^{(n)}$ are bounded for all $n$ ". Conversely, by $(27), y^{(n)}$ can be written in terms of $v^{(n-1)}$ and $y, y^{\prime}, \ldots, y^{(n-1)}$. Thus by induction, " $v^{(n)}$ are bounded for all $n$ " implies " $y^{(n)}$ are bounded for all $n$ ".

\section{Construction of equations exhibiting resonance.}

We want to construct equations of the form

$$
\varepsilon y^{\prime \prime}-2 x y^{\prime}+g(x, \varepsilon) y=0
$$

where $g$ is analytic with respect to $x, \varepsilon$ in some neighborhood of $0 \in \mathbb{C}^{2}$ such that (28) has resonant solutions and all its formal solutions diverge. It seems that there is no simple example of such an equation. For simplicity, we only treat the case $y_{0}(x) \equiv 1$, which is equivalent to $g(x, 0) \equiv 0$. We will always suppose that $|\varepsilon|<\varepsilon_{0}$ with some fixed $\varepsilon_{0} \leq 1$. The advantage of having a fixed coefficient of $y^{\prime}$ is the following: If $y_{1}$ is a solution of (28), then a second solution is given by $y_{2}=T y_{1}$, where

$$
(T y)(x, \varepsilon)=y(x, \varepsilon) \int_{0}^{x} e^{t^{2} / \varepsilon} y(t, \varepsilon)^{-2} d t
$$

The method of construction is as follows.

1. We construct a function $z: D \times S \rightarrow \mathbb{C}$, where $D=D(0,1) \subset \mathbb{C}$ is the open unit disk and $S$ is the open sector $S\left(-\frac{5 \pi}{4}, \frac{5 \pi}{4}, \varepsilon_{0}\right)$ on the Riemann surface of the logarithm, that has the following properties

$$
z(0, \varepsilon) \equiv 1 \text { and } z(x, \varepsilon) \rightarrow 1 \text { as } S \ni \varepsilon \rightarrow 0
$$

uniformly on $D$ and

$$
z\left(x, \varepsilon e^{2 \pi i}\right)=z(x, \varepsilon)+e^{2 / \varepsilon}(T z)(x, \varepsilon) \text { for }|\arg \varepsilon+\pi|<\frac{\pi}{4},
$$

where $T$ is the operator defined by $(29)$. 
2. We show that the equation (28) satisfied by $z$, i.e. the one with

$$
g(x, \varepsilon)=-\varepsilon \frac{z^{\prime \prime}}{z}(x, \varepsilon)+2 x \frac{z^{\prime}}{z}(x, \varepsilon)
$$

is a non trivial example of an equation having an analytic resonant solution, in the sense that the only convergent formal solution is the zero solution.

Briefly, we construct an equation (28) such that its analytic resonant solution has a prescribed monodromy when $\varepsilon$ surrounds the origin once.

Remarks: 1 . The constant function $y_{0}(x) \equiv 1$ can be replaced by any function that does not vanish on the closure of $D$. The coefficient $2 x$ of $y^{\prime}$ can be replaced by other functions of $x$ or $(x, \varepsilon)$; this is particularly interesting in the case $x^{p}$ with odd $p$.

2. The function $e^{2 / \varepsilon}$ can be replaced by any function analytic in the sector $|\arg \varepsilon+\pi|<$ $\frac{\pi}{4}$ that is exponentially small as $\varepsilon \rightarrow 0$. The sector $S$ can be replaced by any good covering. The size of the $x$-neighborhood on which $z$ is constructed depends on the constant (here 2 ) in the exponent and on the good covering. This allows the construction of resonant equations on any convex $x$-domain containing 0 .

3. The above function $z$ has not necessarily real values for real $x, \varepsilon$, but this can be accomplished by using a more "symmetric" monodromy relation, for example

$$
z\left(x, \varepsilon e^{\pi i}\right)-i e^{-2 / \varepsilon}(T z)\left(x, \varepsilon e^{\pi i}\right)=z\left(x, \varepsilon e^{-\pi i}\right)+i e^{-2 / \varepsilon}(T z)\left(x, \varepsilon e^{-\pi i}\right) \text { for } \quad|\arg \varepsilon|<\frac{\pi}{4} .
$$

The first part of the above outline is rather technical and involved. We will present the details at the end of this section (theorem 19). In part 2, we have to show that $g$ mentioned there is analytic in $x, \varepsilon$ and that all non zero formal solutions of the corresponding equation diverge. The fact that $z$ is not a single valued function of $\varepsilon$ because of (31) already indicates that if it has an asymptotic expansion as $\varepsilon \rightarrow 0$ it will not be convergent.

Suppose we have already shown the existence of a function $z$ satisfying (30) and (31). Reducing $\varepsilon_{0}$ if necessary, we can define a function $g: D \times S \rightarrow \mathbb{C}$ by (32). As $g$ remains bounded as $\varepsilon \rightarrow 0$ the analyticity of $g$ follows from

Proposition 18. - The function $g$ defined by (32) is single valued, i.e. $g\left(x, \varepsilon e^{2 \pi i}\right)=$ $g(x, \varepsilon)$ for $x \in D, \varepsilon \in S,|\arg \varepsilon+\pi|<\frac{\pi}{4}$.

Proof. This could be verified by a short calculation, but there is another way of proof. Let us define $g$ by (32). Then $z$ satisfies (28) with this function $g$. We have seen that $T z$ satisfies the same equation $z$ does, hence the right hand side of (31) also does. Using (31), we obtain that $z(x, \varepsilon)$ and $z\left(x, \varepsilon e^{2 \pi i}\right)$ satisfy the same equation (28) for $x \in D$ and $|\arg \varepsilon+\pi|<\frac{\pi}{4}$. Using again (32), the proof is complete.

As $|(T z)(x, \varepsilon)| \leq C e^{|x|^{2} /|\varepsilon|}$ for $x \in D,|\arg \varepsilon+\pi|<\frac{\pi}{4}$, the difference $z\left(x, \varepsilon e^{2 \pi i}\right)-z(x, \varepsilon)$ is exponentially small for $x \in D$. By a theorem of Ramis-Sibuya (see below, too), $z(x, \varepsilon)$ has an asymptotic expansion of Gevrey order 1 as $\varepsilon \rightarrow 0$. We denote by $\hat{z}(x, \varepsilon)=$ $\sum_{n=0}^{\infty} z_{n}(x) \varepsilon^{n}$ the corresponding formal series.

As $z$ is not single valued, the series $\hat{z}$ necessarily diverges for some values of $x \neq 0$. In the sequel, we will even determine the asymptotic behavior of $z_{n}(x)$ as $n \rightarrow \infty$ for $x \neq 0$, $\arg x \notin \frac{\pi}{4} \mathbb{Z}$. 
Because of (30), we have as arg $\varepsilon= \pm \pi, \varepsilon \rightarrow 0$

$$
T z(x, \varepsilon) \sim \frac{1}{2} \sqrt{-\pi \varepsilon} \text { if }|\arg x|<\frac{\pi}{4} \text { or }|\arg x-\pi|<\frac{\pi}{4},
$$

and

$$
T z(x, \varepsilon) \sim \frac{\varepsilon}{2 x} e^{x^{2} / \varepsilon} \text { if }|\arg x \pm \pi / 2|<\frac{\pi}{4} .
$$

Of course, these approximations are only uniform with respect to $x$ in compact subsets of the above sectors. This implies

$$
z\left(x, \varepsilon e^{2 \pi i}\right)-z(x, \varepsilon) \sim \frac{1}{2} \sqrt{-\pi \varepsilon} e^{2 / \varepsilon} \quad \operatorname{resp} . \quad z\left(x, \varepsilon e^{2 \pi i}\right)-z(x, \varepsilon) \sim \frac{\varepsilon}{2 x} \exp \left(\frac{2+x^{2}}{\varepsilon}\right)
$$

in the above cases. Using the Cauchy-Heine formula [19]

$$
z(x, \varepsilon)=\frac{1}{2 \pi i} \int_{\varepsilon_{0} e^{-\pi i}}^{\varepsilon_{0} e^{\pi i}} z(x, \mu) \frac{d \mu}{\mu-\varepsilon}+\frac{1}{2 \pi i} \int_{0}^{\varepsilon_{0} e^{-\pi i}}\left(z(x, \mu)-z\left(x, \mu e^{2 \pi i}\right)\right) \frac{d \mu}{\mu-\varepsilon}
$$

$\left(x \in D,|\varepsilon|<\varepsilon_{0},|\arg \varepsilon|<\pi\right)$ one obtains

$$
z_{n}(x)=\frac{1}{2 \pi i} \int_{\varepsilon_{0} e^{-\pi i}}^{\varepsilon_{0} e^{\pi i}} z(x, \mu) \mu^{-n} \frac{d \mu}{\mu}+\frac{1}{2 \pi i} \int_{0}^{\varepsilon_{0} e^{-\pi i}}\left(z(x, \mu)-z\left(x, \mu e^{2 \pi i}\right)\right) \mu^{-n} \frac{d \mu}{\mu} .
$$

Using (33), this yields the existence of some constant $C$ and some analytic function $G \neq 0$ such that

$$
z_{n}(x) \sim C 2^{-n} \Gamma\left(n-\frac{1}{2}\right) \text { resp. } z_{n}(x) \sim G(x)\left(2+x^{2}\right)^{-n} \Gamma(n-1)
$$

as $n \rightarrow \infty$ in the above cases.

This confirms the divergent Gevrey 1 character of $\hat{z}$. A priori, it might be possible to construct a convergent series solution by multiplying $\hat{z}$ by a certain formal series independent of $x$. This is not the case, because $\hat{z}(x, \varepsilon)$ is already convergent for $x=0$. Observe that every formal series solution of $(28)$ is necessarily a product of $\hat{z}$ and some formal series independent of $x$. In fact, the Wronskian of two solution is a multiple of $e^{x^{2} / \varepsilon}$ and hence there cannot exist two linearly independent formal solutions.

Therefore, if we have found a function $z$ satisfying (30) and (31), we obtain an example of an analytic equation (28) having an analytic resonant solution such that all non zero formal solutions diverge.

One might think that some related series $\hat{y}\left(t, \varepsilon^{1 / s}\right)=\hat{z}\left(t \varepsilon^{r / s}, \varepsilon\right)$ converges for certain values of $r, s$. This is not the case, however. The derivative $\frac{d \hat{y}}{d t}\left(t, \varepsilon^{1 / s}\right)=\frac{d \hat{z}}{d x}\left(t \varepsilon^{r / s}, \varepsilon\right) \varepsilon^{r / s}$ would have to be convergent, too, but $\frac{d \hat{z}}{d x}(0, \varepsilon)$ diverges. In fact, by $(30)$ and $(31)$, the derivative $\frac{d z}{d x}(0, \varepsilon)$ of $z$ satisfies

$$
\frac{d z}{d x}\left(0, \varepsilon e^{2 \pi i}\right)=\frac{d z}{d x}(0, \varepsilon)+e^{2 / \varepsilon}
$$

for $|\arg \varepsilon+\pi|<\frac{\pi}{4}$. As before, this implies that the coefficients $d_{n}$ of its asymptotic series as $\varepsilon \rightarrow 0$ satisfy $d_{n} \sim C 2^{-n}(n-1)$ ! as $n \rightarrow \infty$, hence it is divergent.

It remains to show 
Theorem 19. - For $\varepsilon_{0}$ sufficiently small, there exists a function $z$ satisfying (30) and (31).

Proof. We put $z(x, \varepsilon)=1+u(x, \varepsilon)$ and it remains to be shown that there exists a function $u: D \times S \rightarrow \mathbb{C}$ such that $u(0, \varepsilon) \equiv 0, u=\mathcal{O}(\varepsilon)$ as $\varepsilon \rightarrow 0$ uniformly for $x \in D$ and

$$
u\left(x, \varepsilon e^{2 \pi i}\right)=u(x, \varepsilon)+e^{2 / \varepsilon}[T(1+u)](x, \varepsilon)
$$

for $x \in D, \varepsilon \in S,|\arg \varepsilon+\pi|<\frac{\pi}{4}$. This will be done by rewriting (34) as a fixed point equation. Thus we introduce two Banach spaces:

- $\mathcal{B}(D)$, the vector space of all functions $v(x, \varepsilon)$ analytic on $D \times V, V=S\left(-\frac{5 \pi}{4},-\frac{3 \pi}{4}, \varepsilon_{0}\right)$, for which there exists some constant $C$ such that $|v(x, \varepsilon)| \leq C|\varepsilon|^{2}$ for $x \in D, \varepsilon \in V$. As norm \|\|$_{2}$ on $\mathcal{B}(D)$, we choose the infimum of these constants.

- $\mathcal{E}(D)$, the vector space of all functions $w(x, \varepsilon)$ analytic on $D \times S, S=S\left(-\frac{5 \pi}{4}, \frac{5 \pi}{4}, \varepsilon_{0}\right)$, for which there exists some constant $K$ such that $|w(x, \varepsilon)| \leq K|\varepsilon|$ for $x \in D, \varepsilon \in S$. As norm \|\|$_{1}$ on $\mathcal{E}(D)$, we choose the infimum of these constants.

For functions $v: V \rightarrow \mathbb{C}$ and $w: S \rightarrow \mathbb{C}$ independent of $x$, we introduce analogously the Banach spaces $\mathcal{B}$ and $\mathcal{E}$.

We use the following lemma that will be proved later.

Lemma 20. - There is a continuous linear operator $\Sigma: \mathcal{B} \rightarrow \mathcal{E}$ such that for every $v \in \mathcal{B}$ its image $w=\Sigma v$ satisfies

$$
w\left(\varepsilon e^{2 \pi i}\right)=w(\varepsilon)+v(\varepsilon) \text { for } \varepsilon \in V .
$$

We define another operator $\Sigma_{D}: \mathcal{B}(D) \rightarrow \mathcal{E}(D)$ by $\left(\Sigma_{D} v\right)(x,)=.\Sigma[v(x,)$.$] for all x \in D$.

Then it is sufficient to show the existence of a fixed point $u$ of $\Sigma_{D} \tilde{T}$ close to $0 \in \mathcal{E}(D)$ satisfying $u(0, \varepsilon) \equiv 0$. Here $\tilde{T}: \mathcal{E}(D) \supset D_{\tilde{T}} \rightarrow \mathcal{B}(D)$ is defined by

$$
(\tilde{T} w)(x, \varepsilon)=e^{2 / \varepsilon}[T(1+w)](x, \varepsilon) \text { for } x \in D, \varepsilon \in V
$$

and $D_{\tilde{T}}$ denotes the subset of $\mathcal{E}(D)$ containing all functions with $|w(x, \varepsilon)| \leq \frac{1}{2}$. The operator $\tilde{T}$ is well defined because $e^{2 / \varepsilon}$ is exponentially small.

As usually, it is shown that $\Sigma_{D} \tilde{T}$ is a contraction for sufficiently small $\varepsilon_{0}$. Thus, it has a fixed point $u \in \mathcal{E}(D)$. Using the definitions of $\tilde{T}$ and $\Sigma_{D}$, we show that $u(0, \varepsilon) \equiv 0$.

Proof of lemma 20. The construction of a holomorphic function with prescribed monodromy is classical. Given $v$ analytic on $V$, the function $w: S\left(-\pi, \pi, \varepsilon_{0}\right) \rightarrow \mathbb{C}$ defined by

$$
w(\varepsilon)=-\frac{1}{2 \pi i} \int_{0}^{-\varepsilon_{0}} v(\mu) \frac{d \mu}{\mu-\varepsilon}
$$

can be continued analytically to an analytic function, also called $w$, on all of $S$ that satisfies (35). Unfortunately, $w$ has a logarithmic singularity at $\varepsilon=-\varepsilon_{0}$ and is not in $\mathcal{E}$. To overcome this difficulty, we consider some average of integrals similarly to (36), roughly speaking, we integrate with respect to $\varepsilon_{0}$. A factor $\varepsilon / \mu$ is introduced to accomodate the norms of $\mathcal{B}$ and $\mathcal{E}$. 
For $v \in \mathcal{B}$, we define $(\Sigma v)(\varepsilon)=\frac{4}{\pi} \int_{-\pi / 8}^{\pi / 8} w_{\theta}(\varepsilon) d \theta$, where $w_{\theta}: S \rightarrow \mathbb{C}$ is defined by

$$
w_{\theta}(\varepsilon)=-\frac{1}{2 \pi i} \int_{0}^{\varepsilon_{\theta}} \frac{\varepsilon}{\mu} v(\mu) \frac{d \mu}{\mu-\varepsilon} \text { for } \varepsilon \in S \text { and } \theta \in[-\pi / 8, \pi / 8] .
$$

Here $\varepsilon_{\theta}=-\varepsilon_{0} \exp (\theta i)$. If $-3 \pi / 4 \leq \arg \varepsilon \leq 3 \pi / 4$ then the path of integration is the line from 0 to $\varepsilon_{\theta}$, if $\arg \varepsilon<-3 \pi / 4$, the path of integration is above $\varepsilon$, if $\arg \varepsilon>3 \pi / 4$, the path of integration is below $\varepsilon$ (see figure 5 ).

Cauchy's formula shows that $w_{\theta}$ satisfies (35) for every $\theta$ and hence so does $w=\Sigma v$. It remains to show that $w \in \mathcal{E}$ and that there exists $K>0$ such that $\|\Sigma v\|_{1} \leq K\|v\|_{2}$ for all $v \in \mathcal{B}$.

Let us first estimate $w_{\theta}(\varepsilon)$. For $-3 \pi / 4 \leq \arg \varepsilon \leq 3 \pi / 4$, we have $|\mu-\varepsilon| \geq|\mu| \sin \frac{\pi}{8}$, hence $\left|\frac{1}{\varepsilon} w_{\theta}(\varepsilon)\right| \leq C_{1}\|v\|_{2}$ with $C_{1}=\frac{\varepsilon_{0}}{2 \pi} \frac{1}{\sin (\pi / 8)}$.

For $-5 \pi / 4<\arg \varepsilon<-3 \pi / 4$, we will show that

$$
\left|\frac{1}{\varepsilon} w_{\theta}(\varepsilon)\right| \leq\left(C_{1}+\varepsilon_{0}+\frac{\varepsilon_{0}}{2 \pi} \int_{\gamma(\theta, \arg \varepsilon+\pi)} \frac{1}{|\mu-\varepsilon|}|d \mu|\right)\|v\|_{2}
$$

with the above $C_{1}$, where $\gamma(\theta, \psi)$ (see figure 5) is the path described by $\mu=\varepsilon_{0} e^{\phi i}$ and $\phi \in\left[-\frac{5 \pi}{4}, \theta-\pi\right]$ in the case $\theta<\psi$, but $\phi \in\left[\theta-\pi,-\frac{3 \pi}{4}\right]$ in the case $\psi \leq \theta$. In fact, if $\theta<\psi=\arg \varepsilon+\pi<\frac{\pi}{4}$ then we can use paths of integration $\tilde{\gamma}$ from 0 to $\varepsilon_{\theta}$ arbitrarily close to $\gamma_{1} \cup \gamma(\theta, \psi)$ (where $\gamma_{1}$ is the line from 0 to $\varepsilon_{0} e^{-5 \pi i / 4}$ ) that are still in the interior of $V$. The estimate

$$
\left|\int_{\tilde{\gamma}} \frac{1}{\mu(\mu-\varepsilon)} v(\mu) d \mu\right| \leq \int_{\tilde{\gamma}}\left|\frac{\mu}{(\mu-\varepsilon)}\right||d \mu|\|v\|_{2}
$$

for all such $\tilde{\gamma}$ and the limit $\tilde{\gamma} \rightarrow \gamma_{1} \cup \gamma(\theta, \psi)$ yield

$$
\left|\frac{1}{\varepsilon} w_{\theta}(\varepsilon)\right| \leq \frac{1}{2 \pi} \int_{\gamma_{1} \cup \gamma(\theta, \psi)} \frac{|\mu|}{|\mu-\varepsilon|}|d \mu|\|v\|_{2} \text {. }
$$

As in the former case, we obtain $\int_{\gamma_{1}} \frac{|\mu|}{|\mu-\varepsilon|}|d \mu| \leq \frac{\varepsilon_{0}}{\sin (\pi / 8)}$. For the remaining integral, it is sufficient to use $|\mu| \leq \varepsilon_{0}$ on $\gamma(\theta, \psi)$. By combining the two integrals, we obtain (38) for $\theta<\arg \varepsilon+\pi$ (without the term $\varepsilon_{0}$ ).
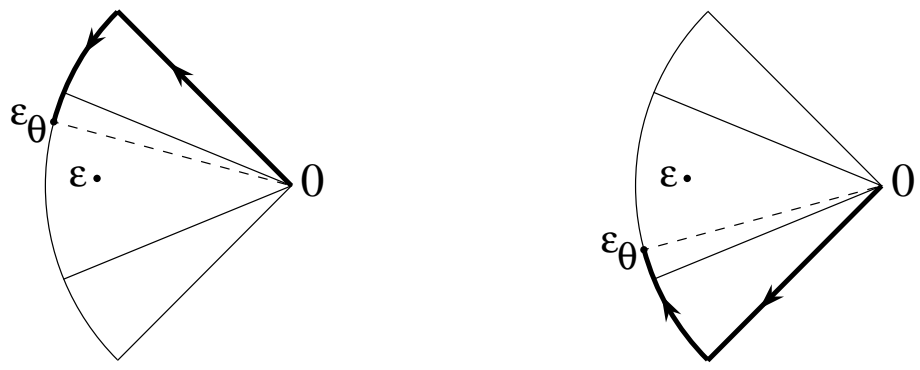

Figure 5: The path $\gamma_{1} \cup \gamma(\theta, \psi)$, on the left in the case $\theta<\psi$, on the right if $\psi \leq \theta$.

In the case $\arg \varepsilon+\pi \leq \theta$, we first use Cauchy's formula which shows

$$
w_{\theta}(\varepsilon)=v(\varepsilon)+\frac{1}{2 \pi i} \int_{0}^{\varepsilon_{\theta}} \frac{\varepsilon}{\mu(\mu-\varepsilon)} v(\mu) d \mu,
$$


where now the path of integration from 0 to $\varepsilon_{\theta}$ is above $\varepsilon$. The estimates of the integral are identical to the above subcase, only there is another term to estimate $\frac{1}{\varepsilon} v(\varepsilon) \leq \varepsilon_{0}\|v\|_{2}$. This proves $(38)$ in the second subcase, too.

For $3 \pi / 4 \leq \arg \varepsilon \leq 5 \pi / 4$, we show analogously

$$
\left|\frac{1}{\varepsilon} w_{\theta}(\varepsilon)\right| \leq\left(C_{1}+\varepsilon_{0}+\frac{\varepsilon_{0}}{2 \pi} \int_{\gamma(\theta, \arg \varepsilon-\pi)} \frac{1}{|\mu-\varepsilon|}|d \mu|\right)\|v\|_{2} .
$$

Recall that $(\Sigma v)(\varepsilon)=\frac{4}{\pi} \int_{-\pi / 8}^{\pi / 8} w_{\theta}(\varepsilon) d \theta$. In order to show that $\Sigma: \mathcal{B} \rightarrow \mathcal{E}$ is continuous, it is hence sufficient to show that

$$
\int_{-\pi / 8}^{\pi / 8} d \theta \int_{\gamma(\theta, \arg \varepsilon+\pi)} \frac{1}{|\mu-\varepsilon|}|d \mu| \leq K
$$

for every $\varepsilon$ with $-5 \pi / 4 \leq \arg \varepsilon \leq-3 \pi / 4,|\varepsilon|<\varepsilon_{0}$. By splitting the exterior integral in two parts at $\theta=\arg \varepsilon+\pi$, if this value is in $[-\pi / 8, \pi / 8]$, it can be seen that the double integral assumes its maximum for $\arg \varepsilon=-\pi$. Thus, it is sufficient to estimate $2 \int_{0}^{\pi / 8} d \theta \int_{\theta}^{\pi / 4} \frac{1}{\left|e^{s i}+\varepsilon / \varepsilon_{0}\right|} d s$ in this case. As the denominator is at least $\sin s$, the theorem of Fubini-Tonelli yields (40) with $K=2 \int_{0}^{\pi / 4} \frac{s}{\sin s} d s$ (by integrating over the triangle $0 \leq \theta \leq s \leq \pi / 4$ instead of the set $0 \leq \theta \leq \pi / 8, \theta \leq s \leq \pi / 4)$.

Remark: Bernard Malgrange, whom we would like to thank here, suggested another proof of lemma 20 using the $\bar{\partial}$ operator. This proof is outlined below.

For convenience, we now think of sectors as subsets of $\mathbb{C}$; thus the directions $d$ and $d+2 \pi$ are identified. We do not indicate the radius of the sectors - it is always $\varepsilon_{0}$. For simplicity, we introduce a second sector $S\left(-\frac{\pi}{4}, \frac{\pi}{4}\right)$.

It is sufficient to show the existence of two analytic functions $w_{1}$ on $S\left(-\frac{\pi}{4}, \frac{5 \pi}{4}\right)$ and $w_{2}$ on $S\left(-\frac{5 \pi}{4}, \frac{\pi}{4}\right)$ such that

$$
w_{1}-w_{2} \equiv v \quad \text { on } \quad S\left(\frac{3 \pi}{4}, \frac{5 \pi}{4}\right)=S\left(-\frac{5 \pi}{4},-\frac{3 \pi}{4}\right) \text { and } w_{1}-w_{2} \equiv 0 \quad \text { on } \quad S\left(-\frac{\pi}{4}, \frac{\pi}{4}\right) .
$$

The idea is to first solve the problem in the class $\mathcal{C}^{\infty}$ and then to add a correction in order to obtain an analytic solution.

Let $\alpha$ be $\mathcal{C}^{\infty}$ on $S\left(\frac{3 \pi}{4}, \frac{5 \pi}{4}\right)$ such that $\alpha \equiv 0$ on $S\left(\frac{3 \pi}{4}, \frac{7 \pi}{8}\right)$ and $\alpha \equiv 1$ on $S\left(\frac{9 \pi}{8}, \frac{5 \pi}{4}\right)$. Then define $\tilde{w}_{1}, \tilde{w}_{2}$ by $\tilde{w}_{1}:=\alpha v, \tilde{w}_{2}:=(\alpha-1) v$ on $S\left(\frac{3 \pi}{4}, \frac{5 \pi}{4}\right)$ and $\tilde{w}_{1}, \tilde{w}_{2}:=0$ on $S\left(-\frac{\pi}{4}, \frac{3 \pi}{4}\right)$, resp. $S\left(-\frac{3 \pi}{4}, \frac{\pi}{4}\right)$. Then conditions $(41)$ are satisfied, but $\tilde{w}_{1}, \tilde{w}_{2}$ are not analytic: We have $\bar{\partial} \tilde{w}_{1}=\bar{\partial} \tilde{w}_{2}=g$ where $g:=\bar{\partial}(\alpha v)=v \bar{\partial} \alpha$ is $C^{\infty}$ on the punctured disk $D\left(0, \varepsilon_{0}\right) \backslash\{0\}$.

Then we construct a $\mathcal{C}^{\infty}$ correction $h$ defined on the punctured disk such that $\bar{\partial} h \equiv g$. This can be done using the classical formula [13]

$$
h(z)=\frac{1}{2 \pi i} \iint_{S\left(\frac{7 \pi}{8}, \frac{9 \pi}{8}\right)} \frac{g(\zeta)}{z-\zeta} d \zeta \wedge d \bar{\zeta} .
$$

The initial problem (41) is then solved by the analytic functions $w_{1}:=\tilde{w}_{1}-h, w_{2}:=\tilde{w}_{2}-h$.

If $\alpha$ is chosen as a function of the angle, say $\alpha\left(r e^{\theta i}\right)=\varphi(\theta)$, then $g\left(\right.$ and hence $\left.w_{1}, w_{2}\right)$ is bounded as soon as $v(z) / z$ is, because $g(z)=v(z) \frac{\partial}{\partial \bar{z}} \alpha(z)=v(z) \frac{i}{\bar{z}} \varphi^{\prime}(\arg z)$. This shows that a bounded linear operator from $\mathcal{B}$ to $\mathcal{E}$ can be constructed in this way. 


\section{References}

[1] R.C. Ackerberg, R.E. O’Malley, Boundary layer Problems Exhibiting Resonance, Studies in Appl. Math., 49 no 3 (1970) 277-295.

[2] E. Benoît, Asymptotic expansions of canards with poles. Application to the stationary unidimensional Schrödinger equation, Bull. Belgian Math. Soc., Supplement "Nonstandard Analysis" (1996) 71-90.

[3] E. Benô̂T, Enlacements de canards, Publications I.H.E.S. 72 (1990) 63-91.

[4] E. Benoît, J.L. Callot, F. Diener, M. Diener, Chasse au canard, Collect. Math., 31, 1-3 (1981) 37-119.

[5] E. Benoît, A. Fruchard, R. Schäfke, G. Wallet, Solutions surstables des équations différentielles complexes lentes-rapides à point tournant, Ann. Fac. Sci. Toulouse, Vol. VII, no 4 (1998) (1-32). .

[6] E. Benoît, A. Fruchard, R. Schäfke, G. Wallet, Overstability : toward a global study, C. R. Acad. Sci. Paris, t. 326, Série I (1998) 873-878.

[7] J.L. Callot, Bifurcation du portrait de phase pour des équations différentielles linéaires du second ordre ayant pour type l'équation d'Hermite, Thèse de Doctoral d'Etat, Strasbourg (1981).

[8] J.L. Callot, Champs lents-rapides complexes à une dimension lente, Ann. Sci. Ec. Norm. Sup., 4e Série, t. 26 (1993) 149-173.

[9] M. Canalis-Durand, J.-P. Ramis, R. Schäfke, Y. Sibuya, Gevrey solutions of singularly perturbed differential equations, A paraître à J. Reine Angew. Math. (1999) http://www-irma.u-strasbg.fr/irma/publications/1999/99017.shtml.

[10] L.P. Cook, W. Eckhaus, Resonance in a boundary value problem of singular perturbation type, Studies in Appl. Math., 52 (1973) 129-139.

[11] P.P.N. De Groen, The nature of resonance in a singular perturbation problem of turning point type, SIAM J. of Math. Anal., 11 (1980), 1-22.

[12] F. Diener, Méthode du plan d'observabilité, Thèse de Doctorat d'Etat, prépublication IRMA, 7, rue René-Descartes, 67084 Strasbourg cedex, France (1981).

[13] L. Hörmander, An introduction to complex analysis in several variables, Elsevier Science B.V., Amsterdam (1966, revised 1973,1990).

[14] N. KopelL, A geometric approach to boundary layer problems exhibiting resonance, SIAM. J. Appl. Math., 37 No2 (1979) 436-458.

[15] W.D. LAKIN, Boundary value problems with a turning point, Studies in Appl. Math., 51 (1972) 261-275.

[16] C.H. LIN, The sufficiency of Matkowsky-condition in the problem of resonance, Trans. Amer. Math. Soc. 278 Nr2 (1983) 647-670. 
[17] B.J. MatKowsky, On boundary layer problems exhibiting resonance, SIAM Review, 17-1 (1975) 82-100.

[18] F.W.J. OLver, Sufficient conditions for Ackerberg-O'Malley resonance, SIAM J. Math. Anal., 9 (1978) 328-355.

[19] Y. SiBUYA, A theorem concerning uniform simplification at a transition point and the problem of resonance, SIAM J. Math. Anal., 12 (1981) 653-668.

[20] W. Wasow, Asymptotic expansions for ordinary differential equations, Interscience, New York (1965).

[21] W. Wasow, Linear Turning Point Theory, Springer, New York (1985).

Augustin Fruchard:

Laboratoire de Mathématiques Calcul Asymptotique

Université de La Rochelle

Pôle Sciences et Technologie

Avenue Michel Crépeau

17042 La Rochelle cedex, FRANCE

e-mail: augustin.fruchard@univ-lr.fr

Reinhard Schäfke:

Département de Mathématiques

Université Louis Pasteur

7 , rue René-Descartes

67084 Strasbourg cedex, FRANCE.

e-mail: schaefke@math.u-strasbg.fr 